\title{
Effective potential and universality in GUT-inspired gauge-Higgs unification
}

\author{
Shuichiro Funatsu $\odot,{ }^{1}$ Hisaki Hatanaka $\odot,{ }^{2}$ Yutaka Hosotani $\odot,{ }^{3}$ Yuta Orikasa $\odot,{ }^{4}$ and Naoki Yamatsu $\odot^{5}$ \\ ${ }^{1}$ Institute of Particle Physics and Key Laboratory of Quark and Lepton Physics (MOE), \\ Central China Normal University, Wuhan, Hubei 430079, China \\ ${ }^{2}$ Osaka, Osaka 536-0014, Japan \\ ${ }^{3}$ Department of Physics, Osaka University, Toyonaka, Osaka 560-0043, Japan \\ ${ }^{4}$ Institute of Experimental and Applied Physics, Czech Technical University in Prague, \\ Husova 240/5, 11000 Prague 1, Czech Republic \\ ${ }^{5}$ Department of Physics, Kyushu University, Fukuoka 819-0395, Japan
}

(Received 28 April 2020; accepted 17 June 2020; published 14 July 2020)

\begin{abstract}
The effective potential for the Aharonov-Bohm phase $\theta_{H}$ in the fifth dimension in GUT-inspired $S O(5) \times U(1) \times S U(3)$ gauge-Higgs unification is evaluated to show that dynamical electroweak symmetry breaking takes place with $\theta_{H} \neq 0$, where the four-dimensional Higgs boson mass of $125 \mathrm{GeV}$ is generated at the quantum level. The cubic and quartic self-couplings $\left(\lambda_{3}, \lambda_{4}\right)$ of the Higgs boson are found to satisfy universal relations, i.e., they are determined, to high accuracy, solely by $\theta_{H}$, irrespective of the values of other parameters in the model. For $\theta_{H}=0.1(0.15), \lambda_{3}$ and $\lambda_{4}$ are smaller than those in the standard model by $7.7 \%(8.1 \%)$ and $30 \%(32 \%)$, respectively.
\end{abstract}

DOI: $10.1103 /$ PhysRevD.102.015005

\section{INTRODUCTION}

The Higgs boson is responsible for the electroweak symmetry breaking in the standard model (SM). The Higgs potential is arranged such that the Higgs field spontaneously develops a nonvanishing vacuum expectation value. Its couplings to quarks and leptons (Yukawa couplings) are determined such that the observed quark-lepton mass spectrum is reproduced. Although the SM seems consistent with almost all experimental data so far obtained, it is yet to be seen whether or not the Higgs boson is exactly what is postulated in the SM. While the gauge sector in the SM is regulated by the gauge principle, the Higgs sector lacks such a principle, which leaves arbitrariness in the theory. The Higgs boson mass acquires large quantum corrections which must be canceled by fine-tuning of parameters in the model.

One approach to overcome these difficulties is gaugeHiggs unification (GHU) in which the four-dimensional (4D) Higgs boson is identified with the 4D fluctuation mode of an Aharonov-Bohm (AB) phase in the fifth dimension (5D). The 4D Higgs field is contained in the extra-dimensional component of gauge potentials. As an AB phase the Higgs boson is massless at the tree level, but acquires a finite mass at the quantum level, independent of

Published by the American Physical Society under the terms of the Creative Commons Attribution 4.0 International license. Further distribution of this work must maintain attribution to the author(s) and the published article's title, journal citation, and DOI. Funded by SCOAP . the cutoff scale and regularization method. The gauge hierarchy problem is naturally solved [1-6].

Recently, substantial advances have been made in gaugeHiggs unification. Realistic models have been constructed which yield nearly the same phenomenology as the SM at low energies and give many predictions to be explored at the LHC and ILC. Most gauge-Higgs unification models are constructed on orbifolds such as $M^{4} \times\left(S^{1} / Z_{2}\right)$ and the Randall-Sundrum (RS) warped space. Chiral fermions naturally emerge on orbifolds [7]. The $S U(2)_{L}$ doublet Higgs field must appear as a zero mode of the fifthdimensional component of gauge fields. This condition leads to gauge groups such as $S U(3) \times U(1)_{X} \times S U(3)_{C}$ or $S O(5) \times U(1)_{X} \times S U(3)_{C}$, among which the latter accommodates the custodial symmetry in the Higgs sector. Quarklepton multiplets are introduced such that with orbifold conditions specified zero modes appear precisely for quarks and leptons, but not for exotic light fermions. They must have observed couplings to $W$ and $Z$ bosons, and their masses must be reproduced. Further, the effective potential for the $\mathrm{AB}$ phase $\theta_{H}$ must have a global minimum at $\theta_{H} \neq 0$ so that the electroweak gauge symmetry is dynamically broken to $U(1)_{\mathrm{EM}}$. As a model satisfying these conditions, $S O(5) \times U(1)_{X} \times S U(3)_{C}$ gauge-Higgs unification is formulated in the RS space [8-20].

In the RS space, which is an anti-de Sitter (AdS) spacetime sandwiched by UV and IR branes, wave functions of dominant components of $W$ and $Z$ bosons are almost constant in the bulk region so that gauge couplings of quarks and leptons are nearly the same as those in 
the SM. The hierarchy between the Kaluza-Klein (KK) mass scale $(\sim 10 \mathrm{TeV})$ and the weak scale $(\sim 100 \mathrm{GeV})$ naturally emerges. Two typical ways of introducing fermions have been investigated. In one type of model (the A model) quarks and leptons are introduced in the vector representation of $S O(5)$. The model predicts large parity violation in the $Z^{\prime}$ couplings of quarks and leptons, which can be checked in the early stage of the ILC experiments with polarized electron and positron beams [14,17,21,22].

It has been noticed, however, that there arises a difficulty in promoting the A model to grand unification [23-27]. The natural extension of the $S O(5) \times U(1)_{X} \times S U(3)_{C}$ model is $S O(11)$ gauge-Higgs grand unification [24]. Up-type quarks are contained in the spinor representation of $S O(11)$, but not in the vector representation so that uptype quarks in the A model do not appear from the $S O(11)$ gauge-Higgs unification. A new way of introducing fermion multiplets has been found which can be embedded into the $S O(11)$ gauge-Higgs grand unification [16]. In this grand unified theory (GUT)-inspired model, or the B model, quarks and leptons are introduced in the spinor and singlet representations of $S O(5)$. It has been shown that quarks and leptons have almost the same gauge couplings as in the SM. Furthermore, the flavor mixing is nicely incorporated with gauge-invariant brane interactions in the $\mathrm{B}$ model. The Cabibbo-Kobayashi-Maskawa (CKM) matrix is obtained, and remarkably flavor-changing neutral-current (FCNC) interactions are naturally suppressed [20].

In this paper we evaluate the effective potential $V_{\text {eff }}\left(\theta_{H}\right)$ in GUT-inspired $S O(5) \times U(1)_{X} \times S U(3)_{C}$ gauge-Higgs unification. It will be shown that with appropriate choices of parameters $V_{\text {eff }}\left(\theta_{H}\right)$ has a global minimum at $\theta_{H} \neq 0$ and the Higgs boson mass $m_{H}=125 \mathrm{GeV}$ is obtained. The cubic and quartic self-couplings of the Higgs boson are determined from $V_{\text {eff }}\left(\theta_{H}\right)$. We shall show that those cubic and quartic self-couplings are, to high accuracy, determined as functions of $\theta_{H}$ only. They do not depend on other parameters of the theory. It will be explained how this property appears in the model.

The effective potential $V_{\text {eff }}\left(\theta_{H}\right)$ is important in discussing phase transitions at finite temperature as well. Recently, a possibility of having first-order phase transitions in gauge-Higgs unification has been argued [28]. At the moment the nature of phase transitions at finite temperature in $S O(5) \times U(1) \times S U(3)$ gauge-Higgs unification remains unclear.

The Higgs boson as an $\mathrm{AB}$ phase in gauge-Higgs unification is similar to that in composite Higgs models in which the Higgs boson appears as a pseudo-NambuGoldstone boson $[9,29,30]$. In both scenarios the Higgs boson field has the character of a phase, but has a quite different mechanism for acquiring its mass. In gauge-Higgs unification the Higgs boson mass is generated by the gauge-invariant dynamics of the $\mathrm{AB}$ phase, whereas it results from the ungauged part of global symmetry in composite Higgs models. Further, in gauge-Higgs unification left-handed and right-handed components of quarks and leptons are normally localized in opposite branes: if lefthanded components are localized near the UV (IR) brane, then right-handed components are localized near the IR (UV) brane. In typical composite Higgs models all light quarks and leptons are assumed to be localized near the UV brane. This leads to big difference in phenomenology associated with $Z^{\prime}$ or technirho bosons. In gauge-Higgs unification in RS space there is large parity violation in $Z^{\prime}$ couplings of quarks and leptons [14,31], whereas such asymmetry is absent in composite Higgs models. GaugeHiggs unification is strictly regulated by the gauge principle.

The paper is organized as follows. In Sec. II the model is introduced. In Sec. III the effective potential $V_{\text {eff }}\left(\theta_{H}\right)$ is evaluated. We show that dynamical electroweak symmetry breaking takes place. The cubic and quartic self-couplings, $\lambda_{3}$ and $\lambda_{4}$, of the Higgs boson are evaluated from $V_{\text {eff }}\left(\theta_{H}\right)$. It is observed there that $\lambda_{3}$ and $\lambda_{4}$ are determined to high accuracy as functions of $\theta_{H}$, irrespective of other parameters in the model. The origin of the $\theta_{H}$ universality in the RS space is clarified in Sec. IV. In Sec. V the spectrum of dark fermions is evaluated. Section VI is devoted to a summary. The mass spectra of all fields in the model are summarized in Appendix A. The functions used for the evaluation of $V_{\text {eff }}\left(\theta_{H}\right)$ are summarized in Appendix B.

\section{MODEL}

The GUT-inspired $S O(5) \times U(1)_{X} \times S U(3)_{C}$ gaugeHiggs unification was introduced in Refs. [16,20]. It is defined in the RS warped space with metric given by [32]

$$
d s^{2}=g_{M N} d x^{M} d x^{N}=e^{-2 \sigma(y)} \eta_{\mu \nu} d x^{\mu} d x^{\nu}+d y^{2},
$$

where $M, N=0,1,2,3,5, \mu, \nu=0,1,2,3, y=x^{5}$, $\eta_{\mu \nu}=\operatorname{diag}(-1,+1,+1,+1), \sigma(y)=\sigma(y+2 L)=\sigma(-y)$, and $\sigma(y)=k y$ for $0 \leq y \leq L$. In terms of the conformal coordinate $z=e^{k y} \quad\left(1 \leq z \leq z_{L}=e^{k L}\right)$ in the region $0 \leq y \leq L$,

$$
d s^{2}=\frac{1}{z^{2}}\left(\eta_{\mu \nu} d x^{\mu} d x^{\nu}+\frac{d z^{2}}{k^{2}}\right) .
$$

The bulk region $0<y<L\left(1<z<z_{L}\right)$ is AdS spacetime with a cosmological constant $\Lambda=-6 k^{2}$, which is sandwiched by the UV brane at $y=0(z=1)$ and the IR brane at $y=L\left(z=z_{L}\right)$. The KK mass scale is $m_{\mathrm{KK}}=$ $\pi k /\left(z_{L}-1\right) \simeq \pi k z_{L}^{-1}$ for $z_{L} \gg 1$.

In addition to the gauge fields $A_{M}^{S U(3)_{C}}, A_{M}^{S O(5)}$, and $A_{M}^{U(1)_{X}}$ of $S U(3)_{C}, S O(5)$, and $U(1)_{X}$, we introduce the matter fields listed in Table I. Fields defined in the bulk satisfy orbifold boundary conditions. Each gauge field satisfies 
TABLE I. The $S U(3)_{C} \times S O(5) \times U(1)_{X}$ content of matter fields is shown in the GUT-inspired B model and previous A model. In the A model only $S U(3)_{C} \times S O(4) \times U(1)_{X}$ symmetry is preserved on the UV brane so that the $S U(2)_{L} \times S U(2)_{R}$ content is shown for brane fields. The B model is analyzed in the present paper.

\begin{tabular}{lcc}
\hline \hline & B model & A model \\
\hline Quark & $\Psi_{(\mathbf{3}, \mathbf{4})}^{\alpha}:(\mathbf{3}, \mathbf{4})_{\frac{1}{6}}, \Psi_{(\mathbf{3}, \mathbf{1})}^{ \pm \alpha}:(\mathbf{3}, \mathbf{1})_{-\frac{1}{3}}^{ \pm}$ & $\Psi_{1}^{\alpha}:(\mathbf{3}, \mathbf{5})_{\frac{2}{3}}, \Psi_{2}^{\alpha}:(\mathbf{3}, \mathbf{5})_{-\frac{1}{3}}$ \\
Lepton & $\Psi_{(\mathbf{1}, \mathbf{4})}^{\alpha}:(\mathbf{1}, \mathbf{4})_{-\frac{1}{2}}$ & $\Psi_{3}^{\alpha}:(\mathbf{1}, \mathbf{5})_{-1} \Psi_{4}^{\alpha}:(\mathbf{1}, \mathbf{5})_{0}$ \\
Dark fermion & $\Psi_{F}^{\beta}:(\mathbf{3}, \mathbf{4})_{\frac{1}{6}}, \Psi_{(\mathbf{1}, \mathbf{5})}^{ \pm \gamma}:(\mathbf{1}, \mathbf{5})_{0}^{ \pm}$ & $\Psi_{F}^{\delta}:(\mathbf{1}, \mathbf{4})_{\frac{1}{2}}$ \\
Brane fermion & $\chi^{\alpha}:(\mathbf{1}, \mathbf{1})_{0}$ & $\hat{\chi}_{1,2,3 R}^{q}:(\mathbf{3},[\mathbf{2}, \mathbf{1}])_{\frac{7}{6}, \frac{1}{6},-\frac{5}{6}}$ \\
& & $\hat{\chi}_{1,2,3 R}^{l}:(\mathbf{1},[\mathbf{2}, \mathbf{1}])_{-\frac{3}{2}, \frac{1}{2},-\frac{1}{2}}$ \\
Brane scalar & $\Phi_{(\mathbf{1}, \mathbf{4})}:(\mathbf{1}, \mathbf{4})_{\frac{1}{2}}$ & $(\mathbf{1},[\mathbf{1}, \mathbf{2}])_{\frac{1}{2}}$ \\
Symmetry of brane interactions & $S U(3)_{C} \times S O(5) \times U(1)_{X}$ & $S U(3)_{C} \times S O(4) \times U(1)_{X}$ \\
\hline \hline
\end{tabular}

$$
\left(\begin{array}{c}
A_{\mu} \\
A_{y}
\end{array}\right)\left(x, y_{j}-y\right)=P_{j}\left(\begin{array}{c}
A_{\mu} \\
-A_{y}
\end{array}\right)\left(x, y_{j}+y\right) P_{j}^{-1}
$$

where $\left(y_{0}, y_{1}\right)=(0, L), P_{0}=P_{1}=I_{3}$ for $A_{M}^{S U(3)_{C}}$, and $P_{0}=P_{1}=1$ for $A_{M}^{U(1)_{X}} . P_{0}=P_{1}=P_{5}^{S O(5)}=\operatorname{diag}\left(I_{4},-1\right)$ for $A_{M}^{S O(5)}$ in the vector representation and $P_{0}=P_{1}=$ $P_{4}^{S O(5)}=\operatorname{diag}\left(I_{2},-I_{2}\right)$ in the spinor representation. Quark and lepton multiplets satisfy

$$
\begin{aligned}
& \Psi_{(\mathbf{3}, \mathbf{4})}^{\alpha}\left(x, y_{j}-y\right)=-P_{\mathbf{4}}^{S O(5)} \gamma^{5} \Psi_{(\mathbf{3}, \mathbf{4})}^{\alpha}\left(x, y_{j}+y\right), \\
& \Psi_{(\mathbf{3}, \mathbf{1})}^{ \pm \alpha}\left(x, y_{j}-y\right)=\mp \gamma^{5} \Psi_{(\mathbf{3}, \mathbf{1})}^{ \pm \alpha}\left(x, y_{j}+y\right), \\
& \Psi_{(\mathbf{1}, \mathbf{4})}^{\alpha}\left(x, y_{j}-y\right)=-P_{\mathbf{4}}^{S O(5)} \gamma^{5} \Psi_{(\mathbf{1 , 4})}^{\alpha}\left(x, y_{j}+y\right),
\end{aligned}
$$

where $\alpha=1 \sim 3$. Dark fermion multiplets satisfy

$$
\begin{aligned}
\Psi_{F}^{\beta}\left(x, y_{j}-y\right) & =(-1)^{j} P_{\mathbf{4}}^{S O(5)} \gamma^{5} \Psi_{F}^{\beta}\left(x, y_{j}+y\right), \\
\Psi_{(\mathbf{1 , 5})}^{ \pm \gamma}\left(x, y_{j}-y\right) & = \pm P_{\mathbf{5}}^{S O(5)} \gamma^{5} \Psi_{(\mathbf{1 , 5})}^{ \pm \gamma}\left(x, y_{j}+y\right),
\end{aligned}
$$

where $\beta=1 \sim N_{F}$ and $\gamma=1 \sim N_{V}$.

The bulk action of each gauge field $\left(A_{M}^{S U(3)_{C}}, A_{M}^{S O(5)}\right.$, or $\left.A_{M}^{U(1)_{X}}\right)$ is given by

$$
\begin{aligned}
S_{\text {bulk }}^{\text {gauge }}= & \int d^{5} x \sqrt{-\operatorname{det} G}\left[-\operatorname{tr}\left(\frac{1}{4} F^{M N} F_{M N}\right.\right. \\
& \left.\left.+\frac{1}{2 \xi}\left(f_{\mathrm{gf}}\right)^{2}+\mathcal{L}_{\mathrm{gh}}\right)\right]
\end{aligned}
$$

where $\sqrt{-\operatorname{det} G}=1 / k z^{5}$ and $F_{M N}=\partial_{M} A_{N}-\partial_{N} A_{M}-$ $i g\left[A_{M}, A_{N}\right]$ with each 5D gauge coupling constant $g$. The gauge fixing $f_{\text {gf }}$ and ghost terms $\mathcal{L}_{\text {gh }}$ have been specified in Ref. [16]. Each fermion multiplet $\Psi(x, y)$ in the bulk has its own bulk-mass parameter $c$ [33]. The covariant derivative is given by

$$
\begin{aligned}
\mathcal{D}(c) & =\gamma^{A} e_{A}{ }^{M}\left(D_{M}+\frac{1}{8} \omega_{M B C}\left[\gamma^{B}, \gamma^{C}\right]\right)-c \sigma^{\prime}(y), \\
D_{M} & =\partial_{M}-i g_{S} A_{M}^{S U(3)}-i g_{A} A_{M}^{S O(5)}-i g_{B} Q_{X} A_{M}^{U(1)} .
\end{aligned}
$$

Here $\sigma^{\prime}=d \sigma(y) / d y$ and $\sigma^{\prime}(y)=k$ for $0<y<L . g_{S}, g_{A}$, and $g_{B}$ are $S U(3)_{C}, S O(5)$, and $U(1)_{X}$ gauge coupling constants. The bulk part of the action for the fermion multiplets, with $\bar{\Psi}=i \Psi^{\dagger} \gamma^{0}$, is given by

$$
\begin{aligned}
S_{\text {bulk }}^{\text {fermion }}= & \int d^{5} x \sqrt{-\operatorname{det} G}\left\{\sum_{J} \bar{\Psi}^{J} \mathcal{D}\left(c_{J}\right) \Psi^{J}\right. \\
& -\sum_{\alpha}\left(m_{D_{\alpha}} \bar{\Psi}_{(\mathbf{3}, \mathbf{1})}^{+\alpha} \Psi_{(\mathbf{3 , 1})}^{-\alpha}+\text { H.c. }\right) \\
& \left.-\sum_{\gamma}\left(m_{V_{\gamma}} \bar{\Psi}_{(\mathbf{1 , 5})}^{+\gamma} \Psi_{(\mathbf{1 , 5})}^{-\gamma}+\text { H.c. }\right)\right\},
\end{aligned}
$$

where the sum $\sum_{J}$ extends over $\Psi^{J}=\Psi_{(\mathbf{3}, \mathbf{4})}^{\alpha}, \Psi_{(\mathbf{1 , 4})}^{\alpha}, \Psi_{(\mathbf{3}, \mathbf{1})}^{ \pm \alpha}$, $\Psi_{F}^{\beta}$, and $\Psi_{(\mathbf{1 , 5})}^{ \pm \gamma}$.

The action for the brane scalar field $\Phi_{(\mathbf{1 , 4})}(x)$ is given by

$$
\begin{aligned}
S_{\text {brane }}^{\Phi}= & \int d^{5} x \sqrt{-\operatorname{det} G} \delta(y) \\
& \times\left\{-\left(D_{\mu} \Phi_{(\mathbf{1}, \mathbf{4})}\right)^{\dagger} D^{\mu} \Phi_{(\mathbf{1}, \mathbf{4})}\right. \\
& \left.-\lambda_{\Phi_{(\mathbf{1 , 4})}}\left(\Phi_{(\mathbf{1 , 4})}^{\dagger} \Phi_{(\mathbf{1 , 4})}-|w|^{2}\right)^{2}\right\},
\end{aligned}
$$

where $D_{\mu}=\partial_{\mu}-i g_{A} A_{\mu}^{S O(5)}-i \frac{1}{2} g_{B} A_{\mu}^{U(1)}$. The action for the gauge-singlet brane fermion $\chi^{\alpha}(x)$ is

$$
S_{\text {brane }}^{\chi}=\int d^{5} x \sqrt{-\operatorname{det} G} \delta(y)\left\{\frac{1}{2} \bar{\chi}^{\alpha} \gamma^{\mu} \partial_{\mu} \chi^{\alpha}-\frac{1}{2} M^{\alpha \beta} \bar{\chi}^{\alpha} \chi^{\beta}\right\} .
$$

$\chi^{\alpha}(x)$ satisfies the Majorana condition $\chi^{c}=\chi$ : 
$\chi=\left(\begin{array}{l}\xi \\ \eta\end{array}\right), \quad \chi^{c}=\left(\begin{array}{c}+\eta^{c} \\ -\xi^{c}\end{array}\right)=e^{i \delta_{C}}\left(\begin{array}{c}+\sigma^{2} \eta^{*} \\ -\sigma^{2} \xi^{*}\end{array}\right)$.

On the UV brane there are $S U(3)_{C} \times S O(5) \times U(1)_{X^{-}}$ invariant brane interactions among the bulk fermion, brane fermion, and brane scalar fields. The relevant parts of the brane interactions are given by

$$
\begin{aligned}
S_{\text {brane }}^{\text {int }}= & -\int d^{5} x \sqrt{-\operatorname{det} G} \delta(y) \\
& \times\left\{\kappa^{\alpha \beta} \bar{\Psi}_{(\mathbf{3}, \mathbf{4})}^{\alpha} \Phi_{(\mathbf{1 , 4})} \cdot \Psi_{(\mathbf{3}, \mathbf{1})}^{+\beta}\right. \\
& \left.+\tilde{\kappa}^{\alpha \beta} \bar{\chi}^{\beta} \tilde{\Phi}_{(\mathbf{1 , 4})}^{\dagger} \Psi_{(\mathbf{1 , 4})}^{\alpha}+\text { H.c. }\right\},
\end{aligned}
$$

where the $\kappa$ 's and $\tilde{\kappa}$ 's are coupling constants and

$$
\Phi_{(\mathbf{1}, \mathbf{4})}=\left(\begin{array}{c}
\Phi_{[\mathbf{2}, \mathbf{1}]} \\
\Phi_{[\mathbf{1}, \mathbf{2}]}
\end{array}\right), \quad \tilde{\Phi}_{(\mathbf{1 , 4})}=\left(\begin{array}{c}
i \sigma^{2} \Phi_{[\mathbf{2}, \mathbf{1}]}^{*} \\
-i \sigma^{2} \Phi_{[\mathbf{1}, \mathbf{2}]}^{*}
\end{array}\right) .
$$

When $\left\langle\Phi_{(\mathbf{1}, \mathbf{4})}\right\rangle=(0,0,0, w)^{t}$, Eq. (2.12) generates additional mass terms:

$$
\int d^{5} x \sqrt{-\operatorname{det} G} \delta(y)\left\{2 \mu^{\alpha \beta} \bar{d}_{R}^{\prime \alpha} D_{L}^{+\beta}+\text { H.c. }\right\}, \quad \mu^{\alpha \beta}=\frac{\kappa^{\alpha \beta} w}{\sqrt{2}}
$$

in the down-type quark sector, and

$-\int d^{5} x \sqrt{-\operatorname{det} G} \delta(y) \frac{m_{B}^{\alpha \beta}}{\sqrt{k}}\left(\bar{\chi}^{\beta} \nu_{R}^{\prime \alpha}+\bar{\nu}_{R}^{\prime \alpha} \chi^{\beta}\right), \quad m_{B}^{\alpha \beta}=\tilde{\kappa}^{\alpha \beta} w \sqrt{k}$

in the neutrino sector. With the Majorana masses in Eq. (2.10), the mass term (2.15) induces the inverse-seesaw mechanism in the neutrino sector [26]. Further, $\left\langle\Phi_{(\mathbf{1 , 4})}\right\rangle \neq 0$ breaks $S O(4) \times U(1)_{X}$ down to $S U(2)_{L} \times U(1)_{Y}$. We assume that $w \gg m_{\mathrm{KK}}$. The $4 \mathrm{D} S U(2)_{L}$ gauge coupling is given by $g_{w}=g_{A} / \sqrt{L}$. The $5 \mathrm{D}$ gauge coupling $g_{Y}^{5 \mathrm{D}}$ of $U(1)_{Y}$ and the $4 \mathrm{D}$ bare Weinberg angle at the tree level, $\theta_{W}^{0}$, are given by

$$
\begin{aligned}
g_{Y}^{5 \mathrm{D}} & =\frac{g_{A} g_{B}}{\sqrt{g_{A}^{2}+g_{B}^{2}}}, \\
\sin \theta_{W}^{0} & =\frac{s_{\phi}}{\sqrt{1+s_{\phi}^{2}}}, \quad s_{\phi}=\frac{g_{B}}{\sqrt{g_{A}^{2}+g_{B}^{2}}} .
\end{aligned}
$$

The bare Weinberg angle $\theta_{W}^{0}$ with a given $\theta_{H}$ is determined to fit the LEP1 data for $e^{+} e^{-} \rightarrow \mu^{+} \mu^{-}$at $\sqrt{s}=m_{Z}$ [34]. Approximately, $\sin ^{2} \theta_{W}^{0} \simeq 0.1140+0.1186 \cos \theta_{H}-$ $0.0014 \cos 2 \theta_{H}$. The values of the gauge couplings turn out to be very close to those in the SM, with $\sin ^{2} \theta_{W}=$ $0.2312[20]$.

The 4D Higgs boson $\Phi_{H}(x)$ is contained in the $S O(5) / S O(4)$ part of $A_{y}^{S O(5)}$. In the $z$ coordinate $A_{z}=$ $(k z)^{-1} A_{y}\left(1 \leq z \leq z_{L}\right)$, and

$$
\begin{aligned}
A_{z}^{(j 5)}(x, z) & =\frac{1}{\sqrt{k}} \phi_{j}(x) u_{H}(z)+\cdots, \\
u_{H}(z) & =\sqrt{\frac{2}{z_{L}^{2}-1} z,} \\
\Phi_{H}(x) & =\frac{1}{\sqrt{2}}\left(\begin{array}{c}
\phi_{2}+i \phi_{1} \\
\phi_{4}-i \phi_{3}
\end{array}\right) .
\end{aligned}
$$

At the quantum level $\Phi_{H}$ develops a nonvanishing expectation value. Without loss of generality, we assume $\left\langle\phi_{1}\right\rangle,\left\langle\phi_{2}\right\rangle,\left\langle\phi_{3}\right\rangle=0$ and $\left\langle\phi_{4}\right\rangle \neq 0$, which is related to the $\mathrm{AB}$ phase $\theta_{H}$ in the fifth dimension. The eigenvalues of

$$
\hat{W}=P \exp \left\{i g_{A} \int_{-L}^{L} d y A_{y}\right\} \cdot P_{1} P_{0}
$$

are gauge invariant. For $A_{y}=(2 k)^{-1 / 2} \phi_{4}(x) v_{H}(y) T^{(45)}$, where $v_{H}(y)=k e^{k y} u_{H}(z)$ for $0 \leq y \leq L$ and $v_{H}(-y)=$ $v_{H}(y)=v_{H}(y+2 L)$, one finds

$$
\begin{aligned}
\hat{W} & =\exp \left\{i f_{H}^{-1} \phi_{4}(x) \cdot 2 T^{(45)}\right\} \\
f_{H} & =\frac{2}{g_{A}} \sqrt{\frac{k}{z_{L}^{2}-1}}=\frac{2}{g_{w}} \sqrt{\frac{k}{L\left(z_{L}^{2}-1\right)}} \\
\theta_{H} & =\frac{\left\langle\phi_{4}\right\rangle}{f_{H}}
\end{aligned}
$$

Note that

$$
A_{z}^{(45)}(x, z)=\frac{1}{\sqrt{k}}\left\{\theta_{H} f_{H}+H(x)\right\} u_{H}(z)+\cdots,
$$

where $H(x)$ is the neutral Higgs boson field. There is a large gauge transformation which shifts $\theta_{H}$ by $2 \pi$, preserving the boundary conditions. The physics is invariant under $\theta_{H} \rightarrow \theta_{H}+2 \pi$. We shall evaluate the effective potential $V_{\text {eff }}\left(\theta_{H}\right)$ in the next section.

\section{EFFECTIVE POTENTIAL}

The effective potential $V_{\text {eff }}\left(\theta_{H}\right)$ at the one-loop level is evaluated from the mass spectra of all fields which depend on $\theta_{H}$. After the Wick rotation into the Euclidean signature it is expressed as 


$$
V_{\mathrm{eff}}\left(\theta_{H}\right)=\sum \pm \frac{1}{2} \int \frac{d^{4} p_{E}}{(2 \pi)^{4}} \sum_{n} \ln \left\{p_{E}^{2}+m_{n}\left(\theta_{H}\right)^{2}\right\},
$$

where the sign $+(-)$ corresponds to bosons (fermions). When the KK spectrum $\left\{m_{n}\left(\theta_{H}\right)\right\}$ is determined by zeros of a function $\rho\left(z ; \theta_{H}\right)$, namely, by

$$
\rho\left(m_{n} ; \theta_{H}\right)=0(n=1,2,3, \ldots),
$$

$V_{\text {eff }}\left(\theta_{H}\right)$ is given [35] by

$$
V_{\mathrm{eff}}\left(\theta_{H}\right)=\sum \pm \frac{1}{(4 \pi)^{2}} \int_{0}^{\infty} d y y^{3} \ln \rho\left(i y ; \theta_{H}\right)
$$

The $\theta_{H}$-dependent part of $V_{\text {eff }}^{\text {1loop }}\left(\theta_{H}\right)$ is finite, independent of the cutoff and regularization method employed.

The spectrum-determining functions $\rho\left(z ; \theta_{H}\right)$ for all fields in the model were given in Ref. [16]. They are summarized in Appendix A for convenience. Relevant contributions come from $W$ and $Z$ gauge fields, top-bottom quark multiplets, and dark fermions in the spinor and vector representations. Contributions from light quarks and leptons are negligible. To avoid unnecessary confusion in the following argument, we denote the effective potential as $V_{\text {eff }}(\theta)$. The physical value $\theta_{H}$ corresponds to the global minimum of $V_{\text {eff }}(\theta)$, namely, $d V_{\text {eff }} /\left.d \theta\right|_{\theta=\theta_{H}}=0$. One finds

The top- and bottom-quark contributions are given by

$$
\begin{aligned}
Q_{\mathrm{top}}^{(2)}(q)= & Q_{\mathrm{bottom}}^{(2)}(q)=0, \\
Q_{\mathrm{top}}^{(1)}(q)= & -\frac{1}{2 \hat{S}_{L}\left(q ; c_{t}\right) \hat{S}_{R}\left(q ; c_{t}\right)+1}, \\
Q_{\mathrm{bottom}}^{(1)}(q)= & -\frac{\widetilde{S_{L} S_{R}}}{2 \hat{S}_{L}\left(q ; c_{t}\right) \hat{S}_{R}\left(q ; c_{t}\right) \widetilde{S_{L} S_{R}}+2|\mu|^{2} \hat{C}_{R}\left(q ; c_{t}\right) \hat{S}_{R}\left(q ; c_{t}\right) \widetilde{C_{L} S_{L}}+1}, \\
\widetilde{S_{L} S_{R}}= & \hat{S}_{L}\left(q ; c_{D_{b}}+\tilde{m}_{D_{b}}\right) \hat{S}_{R}\left(q ; c_{D_{b}}-\tilde{m}_{D_{b}}\right)+\hat{S}_{L}\left(q ; c_{D_{b}}-\tilde{m}_{D_{b}}\right) \hat{S}_{R}\left(q ; c_{D_{b}}+\tilde{m}_{D_{b}}\right) \\
& +\hat{C}_{L}\left(q ; c_{D_{b}}+\tilde{m}_{D_{b}}\right) \hat{C}_{R}\left(q ; c_{D_{b}}-\tilde{m}_{D_{b}}\right)+\hat{C}_{L}\left(q ; c_{D_{b}}-\tilde{m}_{D_{b}}\right) \hat{C}_{R}\left(q ; c_{D_{b}}+\tilde{m}_{D_{b}}\right)-2, \\
\widetilde{C_{L} S_{L}}= & \hat{C}_{L}\left(q ; c_{D_{b}}+\tilde{m}_{D_{b}}\right) \hat{S}_{L}\left(q ; c_{D_{b}}-\tilde{m}_{D_{b}}\right)+\hat{C}_{L}\left(q ; c_{D_{b}}-\tilde{m}_{D_{b}}\right) \hat{S}_{L}\left(q ; c_{D_{b}}+\tilde{m}_{D_{b}}\right) .
\end{aligned}
$$

In the above expressions we have assumed that the brane interaction term (2.12) is diagonal in generation space. $c_{D_{\alpha}}$ is the bulk mass parameter of $\Psi_{(3,1)}^{ \pm \alpha}$ and $\tilde{m}_{D_{\alpha}}=m_{D_{\alpha}} / k$. Numerically, the bottom-quark contribution is very small and may be ignored. There are two kinds of dark fermions: $\Psi_{F}^{\beta}$ and $\Psi_{(1,5)}^{ \pm \gamma}$. Their contributions are given by 


$$
\begin{aligned}
Q_{F}^{(1)}(q)= & \frac{1}{2 \hat{S}_{L}\left(q ; c_{F}\right) \hat{S}_{R}\left(q ; c_{F}\right)+1}, \\
Q_{F}^{(2)}(q)= & 0, \\
Q_{V}^{(1)}(q)= & 0, \\
Q_{V}^{(2)}(q)= & -\frac{2}{\hat{B}_{0}\left(q ; c_{V}, \tilde{m}_{V}\right)}, \\
\hat{B}_{0}\left(q ; c_{V}, \tilde{m}_{V}\right)= & \hat{C}_{L}\left(q ; c_{V}+\tilde{m}_{V}\right) \hat{C}_{R}\left(q ; c_{V}-\tilde{m}_{V}\right) \\
& +\hat{C}_{L}\left(q ; c_{V}-\tilde{m}_{V}\right) \hat{C}_{R}\left(q ; c_{V}+\tilde{m}_{V}\right) \\
& +\hat{S}_{L}\left(q ; c_{V}+\tilde{m}_{V}\right) \hat{S}_{R}\left(q ; c_{V}-\tilde{m}_{V}\right) \\
& +\hat{S}_{L}\left(q ; c_{V}-\tilde{m}_{V}\right) \hat{S}_{R}\left(q ; c_{V}+\tilde{m}_{V}\right) .
\end{aligned}
$$

For the sake of simplicity we set degenerate bulk mass parameters $c_{F}$ for $\Psi_{F}^{\beta}$, and degenerate masses $m_{V}=k \tilde{m}_{V}$ and bulk mass parameters $c_{V}$ for $\Psi_{(1,5)}^{ \pm \gamma}$. We note that contributions from gauge bosons and $\Psi_{(1,5)}^{ \pm \gamma}$ fields to $V_{\text {eff }}(\theta)$ are periodic in $\theta$ with a period $\pi$, whereas those from topand bottom-quark fields and $\Psi_{F}^{\beta}$ fields are periodic with a period $2 \pi$.

The parameters of the model are determined in the following steps. (i) We pick the value of $\theta_{H}$. In other words, we adjust the parameters of the model such that $V_{\text {eff }}(\theta)$ has a global minimum at $\theta=\theta_{H}$. (ii) We take $z_{L}=10^{10}$. Then, $k$ is determined so as to reproduce $m_{Z}$, and the KK mass scale $m_{\mathrm{KK}}=\pi k\left(z_{L}-1\right)^{-1}$ is fixed. (iii) The bulk mass parameters of $\Psi_{(3,4)}^{\alpha}$ and $\Psi_{(1,4)}^{\alpha}$ are fixed from the masses of up-type quarks and charged leptons. In particular, $c_{t}$ is determined by $m_{t}$. (iv) The bulk mass parameters $c_{D_{\alpha}}$ of $\Psi_{(3,1)}^{ \pm \alpha}$ and brane interaction coefficients $\mu^{\alpha \beta}$ are determined so as to reproduce the masses of down-type quarks and the CKM matrix. Similarly, the Majorana mass terms $M^{\alpha \beta}$ and brane interactions $\tilde{\kappa}^{\alpha \beta}$ are determined so as to reproduce neutrino masses and the Pontecorvo-Maki-NakagawaSakata matrix. As remarked above, these parameters are numerically irrelevant for $V_{\text {eff }}(\theta)$. (v) At this stage there remain five parameters to be determined: $\left(n_{F}, c_{F}\right)$ of $\Psi_{F}^{\beta}$ and $\left(n_{V}, c_{V}, \tilde{m}_{V}\right)$ of $\Psi_{(1,5)}^{ \pm \gamma}$. There are two conditions to be satisfied:

$$
\begin{aligned}
& (a):\left.\frac{d V_{\mathrm{eff}}}{d \theta}\right|_{\theta=\theta_{H}}=0, \\
& (b): m_{H}^{2}=\left.\frac{1}{f_{H}^{2}} \frac{d^{2} V_{\mathrm{eff}}}{d \theta^{2}}\right|_{\theta=\theta_{H}},
\end{aligned}
$$

where $m_{H}=125.1 \mathrm{GeV}$. The second condition for the Higgs boson mass $m_{H}$ follows from the fact that the effective potential for the 4D Higgs field $H(x)$ is given by $V_{\text {eff }}\left(\theta_{H}+f_{H}^{-1} H\right)$, as inferred from Eq. (2.20). The conditions (3.8) give two constraints to be satisfied among the five parameters $\left(n_{F}, c_{F}, n_{V}, c_{V}, \tilde{m}_{V}\right)$. We first fix, for instance, $\left(n_{F}, n_{V}, c_{V}\right)$ and determine $\left(c_{F}, \tilde{m}_{V}\right)$ by Eq. (3.8).

One may wonder whether the arbitrary choice of the parameters in the last step diminishes the predictive power of the model. Quite surprisingly, many of the physical quantities do not depend on such details of the parameter choice, being determined solely by $\theta_{H}$. There appears the $\theta_{H}$ universality, which will be explained in the next section.

Here we give some examples. The parameters fixed in steps (i)-(iv) above are tabulated in Table II. In Fig. 1 the effective potential for $\theta_{H}=0.1, n_{F}=n_{V}=2$, and $c_{V}=0$ is displayed. $c_{F}=0.319$ and $\tilde{m}_{V}=0.0806$ are chosen to satisfy Eq. (3.8). One observes that the electroweak symmetry is dynamically broken. In Fig. 2 the contributions of relevant fields to the effective potential $V_{\text {eff }}(\theta)$ are displayed. There is a lower bound for $\theta_{H}$ in order to reproduce the top-quark mass. $\theta_{H} \geq \theta_{c 1}$, where $\theta_{c 1} \sim 0.015$ for $z_{L}=10^{10}$. Similarly, there is a constraint for the warp factor. For $\theta_{H}=0.1, n_{F}=n_{V}=2$, the top-quark mass is reproduced only if $z_{L} \geq z_{L 1} \sim 10^{8.1}$ and dynamical electroweak symmetry breaking is achieved only if $z_{L} \leq z_{L 2} \sim 10^{15.5}$.

The effective potential $V_{\text {eff }}(\theta)$ has more information on the Higgs self-couplings. By expanding $V_{\text {eff }}\left(\theta_{H}+H / f_{H}\right)$, one finds the Higgs self-couplings $\lambda_{n} H^{n}$. The $n$th selfcoupling $\lambda_{n}$ is given by

$$
\left.\lambda_{n} \equiv \frac{1}{n ! f_{H}^{n}} \frac{d^{n} V_{\mathrm{eff}}}{d \theta^{n}}\right|_{\theta=\theta_{H}} .
$$

The couplings $\lambda_{3}$ and $\lambda_{4}$ are plotted in Figs. 3 and 4 as functions of $\theta_{H}$ for $c_{V}=0.2, n_{F}=n_{V}=2$. The fitting curves are given by

$$
\text { B model: } \begin{aligned}
\lambda_{3} / \mathrm{GeV}= & 39.6 \cos \theta_{H}-5.21\left(1+\cos 2 \theta_{H}\right) \\
& -0.00911 \cos 3 \theta_{H}, \\
\lambda_{4}= & -0.0695+0.0852 \cos \theta_{H} \\
& +0.00725 \cos 2 \theta_{H} .
\end{aligned}
$$

$\lambda_{3}$ and $\lambda_{4}$ in the A model are also plotted in Figs. 3 and 4, for which the fitting curves are given by

TABLE II. Parameters determined for $z_{L}=10^{10}$ and $\theta_{H}=0.05$, $0.1,0.15$. We set $\mu^{\alpha \beta}=\mu_{\alpha} \delta_{\alpha \beta}$, and take $c_{D_{b}}=1.04 . \mu_{b}$ is determined so as to reproduce $m_{b}$.

\begin{tabular}{lccccc}
\hline \hline$\theta_{H}$ & $k\left[10^{13} \mathrm{GeV}\right]$ & $m_{\mathrm{KK}}[\mathrm{TeV}]$ & $c_{t}$ & $c_{D_{b}}$ & $\mu_{b}$ \\
\hline 0.05 & 7.68 & 24.1 & -0.226 & 1.04 & 0.106 \\
0.10 & 3.84 & 12.1 & -0.227 & 1.04 & 0.104 \\
0.15 & 2.57 & 8.07 & -0.230 & 1.04 & 0.0990 \\
\hline \hline
\end{tabular}



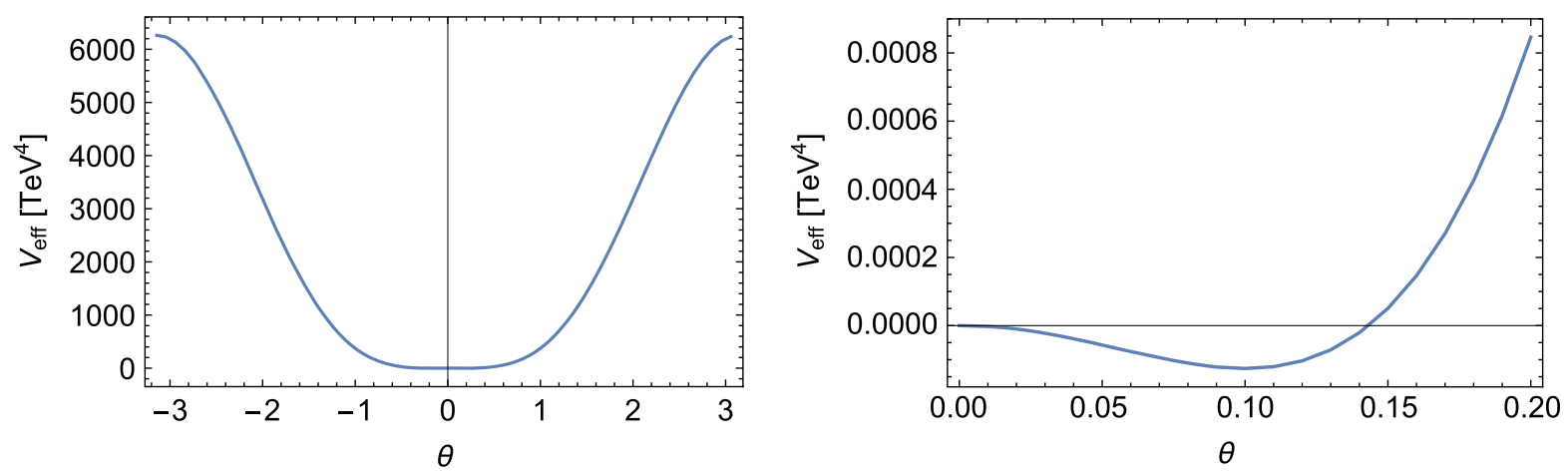

FIG. 1. The effective potential for $\theta_{H}=0.1, n_{F}=2, n_{V}=2$, and $c_{V}=0$. The global minimum is located at $\theta=\theta_{H}$.

A model: $\lambda_{3} / \mathrm{GeV}=32.4 \cos \theta_{H}-2.26\left(1+\cos 2 \theta_{H}\right)$

$$
\begin{aligned}
& -1.1 \cos 3 \theta_{H}, \\
\lambda_{4}= & -0.00264-0.0129 \cos \theta_{H} \\
& +0.0363 \cos 2 \theta_{H} .
\end{aligned}
$$

Note that $\lambda_{3}$ vanishes at $\theta_{H}=\frac{1}{2} \pi$ as a consequence of the $H$ parity in GHU models [36]. For $\theta_{H} \gtrsim 0.6, \lambda_{4}$ becomes negative which, however, does not imply instability. The $\theta$-dependent part of $V_{\text {eff }}(\theta)$ is finite and bounded from below. Gauge-Higgs unification does not experience

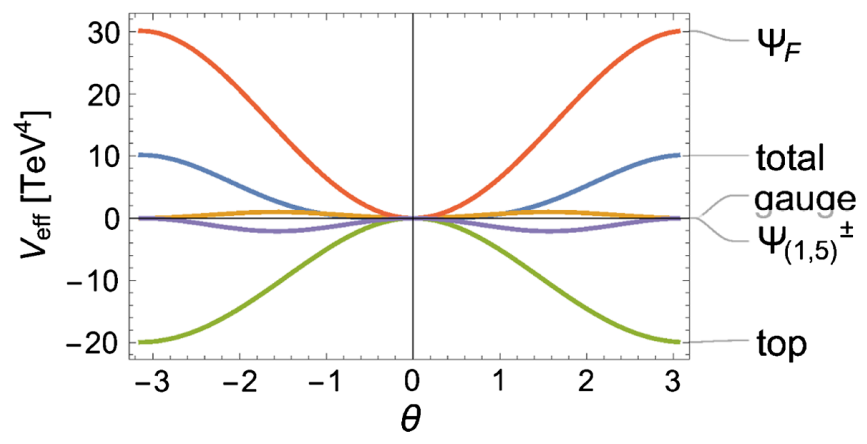

FIG. 2. Contributions of relevant fields to the effective potential for $\theta_{H}=0.1, n_{F}=2, n_{V}=2$, and $c_{V}=0$.

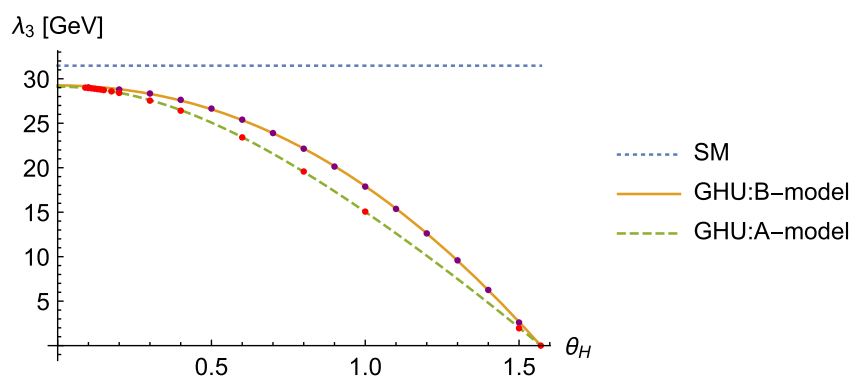

FIG. 3. The cubic coupling $\lambda_{3}$ of the Higgs boson. The fitting curves are given by $\lambda_{3} / \mathrm{GeV}=39.6 \cos \theta_{H}-5.21\left(1+\cos 2 \theta_{H}\right)-$ $0.00911 \cos 3 \theta_{H}$ for the $\mathrm{B}$ model and $\lambda_{3} / \mathrm{GeV}=32.4 \cos \theta_{H}-$ $2.26\left(1+\cos 2 \theta_{H}\right)-1.1 \cos 3 \theta_{H}$ for the A model. The SM value is $\lambda_{3, \mathrm{SM}}=31.5 \mathrm{GeV}$. the vacuum instability problem that afflicts most 4D field theories. From the experimental constraints from LEP1, LEP2, and LHC data for the nonobservation of $Z^{\prime}$ events, it is inferred that $\theta_{H} \lesssim 0.11$. For $\theta_{H} \sim 0.1(0.15), \lambda_{3}$ and $\lambda_{4}$ are smaller than those in the SM by $7.7 \%(8.1 \%)$ and $30 \%$ (32\%), respectively. As explained above, there is a lower bound for $\theta_{H}$ in GHU, namely, $\theta_{H}>\theta_{c 1}$, and there does not exist a $\theta_{H} \rightarrow 0$ limit. It is not surprising that $\lambda_{3}$ and $\lambda_{4}$ deviate from the values in the SM even for small $\theta_{H}$. The couplings $\lambda_{n}(n \geq 5)$ are generated at the one-loop level in both GHU and the SM, which turn out to be finite. The Higgs couplings to quarks, leptons, and $W$ and $Z$ bosons in GHU for small $\theta_{H}$ are very close to those in the SM. There are additional contributions coming from KK modes in GHU. It may be interesting as well to measure these couplings $\lambda_{n}(n \geq 5)$ in future experiments to test predictions from GHU and the SM.

\section{IV. $\theta_{H}$ UNIVERSALITY}

As remarked in the previous section, there remains the arbitrariness in the choice of the parameters in the model. Among the five parameters $\left(n_{F}, c_{F}, n_{V}, c_{V}, \tilde{m}_{V}\right)$ there are only two conditions in Eq. (3.8) that must be obeyed. In the examples given in the previous section, we first fixed $\left(n_{F}, n_{V}, c_{V}\right)$ and determined $\left(c_{F}, \tilde{m}_{V}\right)$ by Eq. (3.8). The Higgs cubic and quartic couplings, $\lambda_{3}$ and $\lambda_{4}$, are

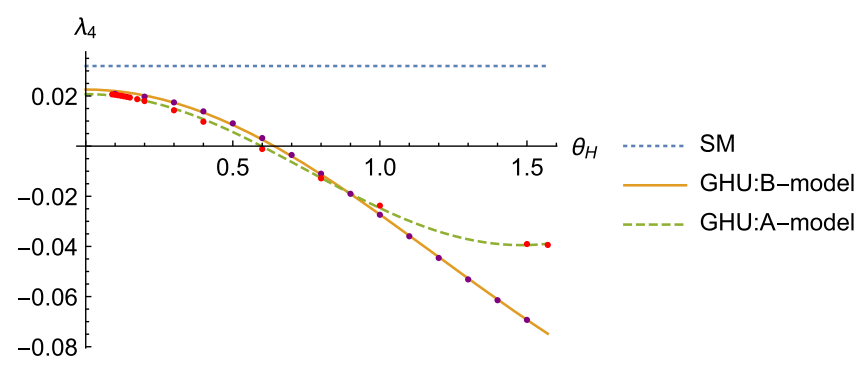

FIG. 4. The quartic coupling $\lambda_{4}$ of the Higgs boson. The fitting curves are given by $\lambda_{4}=-0.0695+0.0852 \cos \theta_{H}+$ $0.00725 \cos 2 \theta_{H}$ for the $\mathrm{B}$ model and $\lambda_{4}=-0.00264-$ $0.0129 \cos \theta_{H}+0.0363 \cos 2 \theta_{H}$ for the A model. The SM value is $\lambda_{4, \mathrm{SM}}=0.0320$. 
evaluated with this choice. One might wonder how $\lambda_{3}$ and $\lambda_{4}$ depend on the choice of the parameters $\left(n_{F}, n_{V}, c_{V}\right)$.

In this section we show that $\lambda_{3}$ and $\lambda_{4}$ are determined, to high accuracy, as functions of $\theta_{H}$ only, and do not depend on the details of the parameter choice. It has been known in GHU that the three-point Higgs couplings to $W, Z$, quarks, and leptons also have the same property [37]. These physical quantities are determined by $\theta_{H}$ to high accuracy. This may be called the $\theta_{H}$ universality. The $\theta_{H}$ universality leads to profound predictive power. Once the value of $\theta_{H}$ is determined by one of the physical quantities, the values of other physical quantities can be predicted.

In Table III we list the values of $\left(\lambda_{3}, \lambda_{4}\right)$ for $\theta_{H}=0.1$ with various choices of $\left(n_{F}, n_{V}, c_{V}\right)$. Although the values of $c_{F}$ and $\tilde{m}_{V}$ depend on the choice of $\left(n_{F}, n_{V}, c_{V}\right)$, the values of $\lambda_{3}$ and $\lambda_{4}$ are universal to high accuracy. $\lambda_{3}$ and $\lambda_{4}$ are determined as functions of $\theta_{H}$ only.

There is a reason for the $\theta_{H}$ universality. We first examine the global behavior of $V_{\text {eff }}(\theta)$ with a given $\theta_{H}$. Notice that the function $A_{p}(\theta)$ in Eq. (3.4) is expanded as

$$
\begin{aligned}
A_{p}(\theta) & =\frac{\left(k z_{L}^{-1}\right)^{4}}{(4 \pi)^{2}} \sum_{\ell=1}^{\infty} \sum_{n=1}^{2} \alpha_{p}^{(n, \ell)} \cos ^{\ell} n \theta, \\
\alpha_{p}^{(n, \ell)} & \equiv \frac{(-1)^{\ell+1}}{\ell} \int_{0}^{\infty} d q q^{3}\left(Q_{p}^{(n)}(q)\right)^{\ell} .
\end{aligned}
$$

As either $Q_{p}^{(1)}(q)$ or $Q_{p}^{(2)}(q)$ for a given $p$ vanishes in Eq. (3.4), $\alpha_{p}^{(1, \ell)}$ or $\alpha_{p}^{(2, \ell)}=0$ for each $p$ in Eq. (4.1).

To understand the qualitative behavior of $V_{\text {eff }}(\theta)$, let us approximate $A_{p}(\theta)$ in Eq. (3.4) by

$$
\begin{aligned}
& \frac{(4 \pi)^{2}}{\left(k z_{L}^{-1}\right)^{4}} A_{p}(\theta) \\
& \quad= \begin{cases}\alpha_{p}^{(2,1)} \cos 2 \theta & \text { for } p=W, Z, S, V, \\
\alpha_{p}^{(1,1)} \cos \theta+\alpha_{p}^{(1,2)} \cos ^{2} \theta & \text { for } p=\text { top, } F .\end{cases}
\end{aligned}
$$

TABLE III. $\theta_{H}$ universality in $\lambda_{3}$ and $\lambda_{4}$ for $\theta_{H}=0.1$ and $z_{L}=10^{10}$. With given $\left(n_{F}, n_{V}, c_{V}\right), c_{F}$ and $\tilde{m}_{V}$ are determined to satisfy the condition (3.8), and $\lambda_{3}$ and $\lambda_{4}$ are evaluated using Eq. (3.9).

\begin{tabular}{lclcrcc}
\hline \hline$n_{F}$ & $n_{V}$ & $c_{V}$ & \multicolumn{1}{c}{$c_{F}$} & \multicolumn{1}{c}{$\tilde{m}_{V}$} & $\lambda_{3}(\mathrm{GeV})$ & \multicolumn{1}{c}{$\lambda_{4}$} \\
\hline 2 & 2 & 0. & 0.319 & 0.0806 & 29.03 & 0.02083 \\
2 & 2 & 0.2 & 0.319 & 0.0777 & 29.03 & 0.02083 \\
2 & 2 & 0.5 & 0.322 & -0.0371 & 29.02 & 0.02078 \\
4 & 2 & 0. & 0.425 & 0.0794 & 29.02 & 0.02082 \\
4 & 2 & 0.2 & 0.425 & 0.0765 & 29.02 & 0.02082 \\
4 & 2 & 0.5 & 0.426 & -0.0350 & 29.01 & 0.02076 \\
2 & 4 & 0. & 0.318 & 0.0964 & 29.03 & 0.02084 \\
2 & 4 & 0.2 & 0.318 & 0.0937 & 29.03 & 0.02084 \\
2 & 4 & 0.5 & 0.319 & 0.0615 & 29.03 & 0.02083 \\
\hline \hline
\end{tabular}

Note that $\left|\alpha_{p}^{(n, 2)} / \alpha_{p}^{(n, 1)}\right|<0.05$. As the contributions from $p=$ top, $F$ are 1 order of magnitude larger than those from $p=W, Z, S, V$, the $\cos ^{2} \theta$ terms have been retained for the top and $F . V_{\text {eff }}(\theta)$ in this approximation, denoted as $V_{\text {app }}(\theta)$, is given by

$$
\begin{aligned}
V_{\text {app }}(\theta) & =\frac{\left(k z_{L}^{-1}\right)^{4}}{(4 \pi)^{2}}\left(-B_{1} \cos \theta+B_{2} \cos 2 \theta\right), \\
B_{1} & =12 \alpha_{\text {top }}^{(1,1)}+12 n_{F} \alpha_{F}^{(1,1)}, \\
B_{2} & =\alpha_{\text {gauge }}-8 n_{V} \alpha_{V}^{(2,1)}-6 \alpha_{\mathrm{top}}^{(1,2)}-6 n_{F} \alpha_{F}^{(1,2)}, \\
\alpha_{\text {gauge }} & =2\left(3-\xi^{2}\right) \alpha_{W}^{(2,1)}+\left(3-\xi^{2}\right) \alpha_{Z}^{(2,1)}+3 \xi^{2} \alpha_{S}^{(2,1)} .
\end{aligned}
$$

The condition (a) in Eq. (3.8) leads to $B_{1}=4 B_{2} \cos \theta_{H}$. Then, the condition $(b)$ in Eq. (3.8) implies that

$$
B_{2} \sim \frac{16 \pi^{2}}{g_{w}^{2}(k L)^{2}}\left(\frac{m_{H}}{m_{W}}\right)^{2},
$$

where the relations $m_{\mathrm{KK}} \sim \pi k z_{L}^{-1}, m_{W} \sim(k / L)^{1 / 2} z_{L}^{-1} \sin \theta_{H}$, and $f_{H} \sim 2 m_{W} / g_{w} \sin \theta_{H}$ have been used. It follows that

$$
\begin{aligned}
V_{\text {app }}(\theta) & =V_{0} u(\theta), \\
u(\theta) & =-4 \cos \theta_{H} \cos \theta+\cos 2 \theta, \\
V_{0} & =\frac{m_{W}^{2} m_{H}^{2}}{g_{w}^{2} \sin ^{4} \theta_{H}} .
\end{aligned}
$$

The cubic and quartic Higgs self-couplings are given by

$$
\begin{aligned}
& \lambda_{3}^{\text {app }} \sim \frac{g_{w} m_{H}^{2}}{4 m_{W}} \cos \theta_{H}, \\
& \lambda_{4}^{\text {app }} \sim \frac{g_{w}^{2} m_{H}^{2}}{96 m_{W}^{2}}\left(7 \cos ^{2} \theta_{H}-4\right) .
\end{aligned}
$$

The approximate formulas (4.5) and (4.6) represent the qualitative behavior of the effective potential $V_{\text {eff }}(\theta)$, but exhibit a slight deviation from the values in Table III and the fitting curves (3.10) and (3.11). We first note that the form of $V_{\text {app }}(\theta)$ is fixed, once one makes the ansatz that $V_{\text {eff }}(\theta)$ is expressed in terms of two functions: $\cos \theta$ and $\cos 2 \theta$. The quantities relevant for determining $\lambda_{3}$ and $\lambda_{4}$ are $B_{1}$ and $B_{2}$, but not detailed values of the parameters in the models considered. In the $A$ or $B$ model the same universality relations (4.6) result in this approximation. It is easy to confirm that the formulas (4.6) reproduce the $\mathrm{SM}$ values at $\theta_{H}=0$,

$$
\left.\lambda_{3}^{\mathrm{app}}\right|_{\theta_{H}=0}=\lambda_{3, \mathrm{SM}},\left.\quad \lambda_{4}^{\mathrm{app}}\right|_{\theta_{H}=0}=\lambda_{4, \mathrm{SM}} .
$$

We also note that $u(\pi)-u(0)=8 \cos \theta_{H}$ and $u\left(\theta_{H}\right)-u(0)=$ $-2\left(1-\cos \theta_{H}\right)^{2}$. For small $\theta_{H}, u(\pi)-u(0) \sim 8$ and 
$u\left(\theta_{H}\right)-u(0) \sim-\frac{1}{2} \theta_{H}^{4}$, which explains the behavior of $V_{\text {eff }}(\theta)$ for $\theta_{H}=0.1$ seen in Fig. 1.

To understand the $\theta_{H}$ universality demonstrated in the previous section we must refine our arguments. The universality was first found in the A model of $S O(5) \times$ $U(1)$ gauge-Higgs unification [13]. The mechanism that yields the $\theta_{H}$ universality was explained in Ref. [38]. We generalize the argument for the current $\mathrm{B}$ model. The important observation is that $\lambda_{3}$ and $\lambda_{4}$ are determined by the local behavior of the effective potential $V_{\text {eff }}\left(\theta_{H}\right)$ in the vicinity of the global minimum at $\theta=\theta_{H}$, and the universality reflects the local—but not global—behavior of $V_{\text {eff }}\left(\theta_{H}\right)$.

The effective potential $V_{\text {eff }}(\theta)$ [Eq. (3.4)] is decomposed into three parts:

$$
\begin{aligned}
V_{\text {eff }}(\theta)= & \frac{\left(k z_{L}^{-1}\right)^{4}}{(4 \pi)^{2}}\left\{h_{0}(\theta)+n_{F} h_{F}\left(\theta ; c_{F}, z_{L}\right)\right. \\
& \left.+n_{V} h_{V}\left(\theta ; c_{V}, \tilde{m}_{V}, z_{L}\right)\right\},
\end{aligned}
$$

where $h_{0}(\theta)$ represents the contributions from gauge and top-quark fields. With $\theta_{H}, z_{L}$, and $\xi$ specified, $k$ is determined from $m_{Z}$ and $c_{t}$ is subsequently determined from $m_{t}$, so that $h_{0}(\theta)$ is fixed. All other parameters associated with quarks and leptons are irrelevant for
$V_{\text {eff }}(\theta)$. There remain five parameters $\left(n_{F}, c_{F}, n_{V}, c_{V}, \tilde{m}_{V}\right)$ to be specified in Eq. (4.8). They must be adjusted such that the two conditions in Eq. (3.8) are satisfied. The important feature in the RS space with $z_{L} \gg 1$ is that the $\theta$ dependence of $h_{F}\left(\theta ; c_{F}, z_{L}\right)$ and $h_{V}\left(\theta ; c_{V}, \tilde{m}_{V}, z_{L}\right)$ factorizes near $\theta=\theta_{H}$,

$$
\begin{aligned}
h_{F}\left(\theta ; c_{F}, z_{L}\right) & \simeq \alpha_{F}\left(c_{F}, z_{L}\right) \tilde{h}_{F}(\theta), \\
h_{V}\left(\theta ; c_{V}, \tilde{m}_{V}, z_{L}\right) & \simeq \alpha_{V}\left(c_{V}, \tilde{m}_{V}, z_{L}\right) \tilde{h}_{V}(\theta),
\end{aligned}
$$

to very high accuracy. This can be confirmed numerically from the formula for $A_{p}(\theta)$ in Eq. (3.4). The relation (4.9) implies, for instance, that the ratio $h_{F}\left(\theta ; c_{F}^{(1)}, z_{L}\right) /$ $h_{F}\left(\theta ; c_{F}^{(2)}, z_{L}\right)$ is $\theta$ independent near $\theta_{H}$. For $\theta_{H}=0.1$, $z_{L}=10^{10}$, and $\left(c_{F}^{(1)}, c_{F}^{(2)}\right)=(0.3,0.4)$, the ratio varies from 1.7916 to 1.7896 in the range $0.09 \leq \theta \leq 0.11$. The variation is only $0.1 \%$. We stress that these factorization formulas are valid only locally, namely, near $\theta=\theta_{H}$, and $\tilde{h}_{F}(\theta)$ and $\tilde{h}_{V}(\theta)$ depend on $\theta_{H}$. The $z_{L}$ dependence of $h_{F}\left(\theta ; c_{F}, z_{L}\right)$ and $h_{V}\left(\theta ; c_{V}, \tilde{m}_{V}, z_{L}\right)$ is also tiny in the range $10^{8} \lesssim z_{L} \lesssim 10^{15}$.

Let us pick a set of values $\left(n_{F}, n_{V}, c_{V}\right)$ and determine $\left(c_{F}, \tilde{m}_{V}\right)$ by Eq. (3.8). Making use of Eq. (4.9), one finds

$$
\begin{gathered}
{\left[\frac{d h_{0}}{d \theta}+n_{F} \alpha_{F}\left(c_{F}, z_{L}\right) \frac{d \tilde{h}_{F}}{d \theta}+n_{V} \alpha_{V}\left(c_{V}, \tilde{m}_{V}, z_{L}\right) \frac{d \tilde{h}_{V}}{d \theta}\right]_{\theta=\theta_{H}}=0} \\
{\left[\frac{d^{2} h_{0}}{d \theta^{2}}+n_{F} \alpha_{F}\left(c_{F}, z_{L}\right) \frac{d^{2} \tilde{h}_{F}}{d \theta^{2}}+n_{V} \alpha_{V}\left(c_{V}, \tilde{m}_{V}, z_{L}\right) \frac{d^{2} \tilde{h}_{V}}{d \theta^{2}}\right]_{\theta=\theta_{H}}=\frac{(4 \pi)^{2} m_{H}^{2} f_{H}^{2}}{\left(k z_{L}^{-1}\right)^{4}}}
\end{gathered}
$$

We perform this procedure for two sets: $\left(n_{F}, n_{V}, c_{V}\right)=$ $\left(n_{F}^{(1)}, n_{V}^{(1)}, c_{V}^{(1)}\right)$ and $\left(n_{F}^{(2)}, n_{V}^{(2)}, c_{V}^{(2)}\right)$. Then, Eq. (4.10) implies that

$$
\begin{aligned}
n_{F}^{(1)} \alpha_{F}\left(c_{F}^{(1)}, z_{L}\right) & =n_{F}^{(2)} \alpha_{F}\left(c_{F}^{(2)}, z_{L}\right) \equiv \beta_{F}, \\
n_{V}^{(1)} \alpha_{V}\left(c_{V}^{(1)}, \tilde{m}_{V}^{(1)}, z_{L}\right) & =n_{V}^{(2)} \alpha_{V}\left(c_{V}^{(2)}, \tilde{m}_{V}^{(2)}, z_{L}\right) \equiv \beta_{V} .
\end{aligned}
$$

Although the values of $\left(c_{F}, \tilde{m}_{V}\right)$ depend on the choice of $\left(n_{F}, n_{V}, c_{V}\right), \beta_{F}=n_{F} \alpha_{F}\left(c_{F}, z_{L}\right)$ and $\beta_{V}=n_{V} \alpha_{V}\left(c_{V}, \tilde{m}_{V}, z_{L}\right)$ are universal, provided solutions exist. Consequently, one obtains

$$
\begin{aligned}
V_{\mathrm{eff}}(\theta) & \simeq \frac{\left(k z_{L}^{-1}\right)^{4}}{(4 \pi)^{2}} \bar{h}(\theta), \\
\bar{h}(\theta) & =h_{0}(\theta)+\beta_{F} \tilde{h}_{F}(\theta)+\beta_{V} \tilde{h}_{V}(\theta) .
\end{aligned}
$$

It immediately follows that

$$
\begin{aligned}
& \lambda_{3}\left(\theta_{H}\right)=\frac{g_{w} m_{H}^{2} \sin \theta_{H}}{12 m_{W}} \frac{\bar{h}^{(3)}\left(\theta_{H}\right)}{\bar{h}^{(2)}\left(\theta_{H}\right)}, \\
& \lambda_{4}\left(\theta_{H}\right)=\frac{g_{w}^{2} m_{H}^{2} \sin ^{2} \theta_{H}}{96 m_{W}^{2}} \frac{\bar{h}^{(4)}\left(\theta_{H}\right)}{\bar{h}^{(2)}\left(\theta_{H}\right)},
\end{aligned}
$$

which explains the $\theta_{H}$ universality observed in the previous section. The relevant quantities for $\lambda_{3}$ and $\lambda_{4}$ are $\beta_{F}\left(\theta_{H}\right)$ and $\beta_{V}\left(\theta_{H}\right)$, but not $\left(n_{F}, n_{V}, c_{V}\right)$. As mentioned above, the $z_{L}$ dependence of $h_{F}\left(\theta ; c_{F}, z_{L}\right)$ and $h_{V}\left(\theta ; c_{V}\right.$, $\left.\tilde{m}_{V}, z_{L}\right)$ is weak. The $\theta_{H}$ universality stays valid to good approximation even for varying $z_{L}$. For instance, for $\theta_{H}=0.1$ and $\left(n_{F}, n_{V}, c_{V}\right)=(2,2,0)$, the resultant $\left(\lambda_{3}, \lambda_{4}\right)$ are $(28.93 \mathrm{GeV}, 0.02042)$ for $z_{L}=1.237 \times 10^{8}$, which should be compared with $(29.03 \mathrm{GeV}, 0.02083)$ for $z_{L}=10^{10}$.

The $\theta_{H}$ universality is observed in other physical quantities. The Higgs boson couplings $g_{W W H}$ and $g_{\mathrm{ZZH}}$ 
TABLE IV. KK mass and dark fermion masses are shown in units of TeV for various $\theta_{H}$ with $z_{L}=10^{10}, n_{F}=n_{V}=2$, and $c_{V}=0.2$. Charged and neutral components of $\Psi_{(1,5)}^{ \pm}$have nearly the same masses.

\begin{tabular}{lccc}
\hline \hline$\theta_{H}$ & $m_{K K}$ & $\Psi_{F}$ & $\Psi_{(1,5)}^{ \pm}$ \\
\hline 0.05 & 24.1 & 6.30 & 5.60 \\
0.10 & 12.1 & 3.42 & 2.84 \\
0.15 & 8.07 & 2.38 & 1.91 \\
0.20 & 6.08 & 1.82 & 1.45 \\
\hline \hline
\end{tabular}

to $W$ and $Z$, and Yukawa couplings $y_{f}$ to quarks and leptons are given, to good approximation, by $[20,37]$

$$
\begin{aligned}
g_{W W H} & =g_{w} m_{W} \cos \theta_{H}, \\
g_{\mathrm{ZZH}} & =\frac{g_{w} m_{Z}}{\cos \theta_{W}^{0}} \cos \theta_{H}, \\
y_{f} & = \begin{cases}\frac{m_{f}}{v_{\mathrm{SM}}} \cos \theta_{H} & \text { in the A model, } \\
\frac{m_{f}}{v_{\mathrm{SM}}} \cos ^{2} \frac{1}{2} \theta_{H} & \text { in the B model, }\end{cases}
\end{aligned}
$$

where $v_{\mathrm{SM}}=f_{H} \sin \theta_{H}=2 m_{W} / g_{w}$. For small $\theta_{H}$, the deviations of the Higgs couplings in Eq. (4.14) are small, whereas the deviations of $\lambda_{3}$ and $\lambda_{4}$ become substantial. We remark that the relations in Eq. (4.14) have been derived in the composite Higgs model where the parameter $\sqrt{\xi}=v / f$ corresponds to $\theta_{H}$ in GHU $[29,30]$.

\section{DARK FERMIONS}

Although the $\theta_{H}$ universality holds for various couplings associated with the Higgs boson, the masses of dark fermions $\Psi_{F}$ and $\Psi_{(1,5)}^{ \pm}$, for instance, sensitively depend on the choice of the parameters $\left(n_{F}, n_{V}, c_{V}\right)$. They are determined by Eq. (A12) for $\Psi_{F}$ and Eqs. (A13) and (A14) for charged and neutral components of $\Psi_{(1,5)}^{ \pm}$. In Table IV we list their masses for various $\theta_{H}$ with $n_{F}=n_{V}=2$. Dark

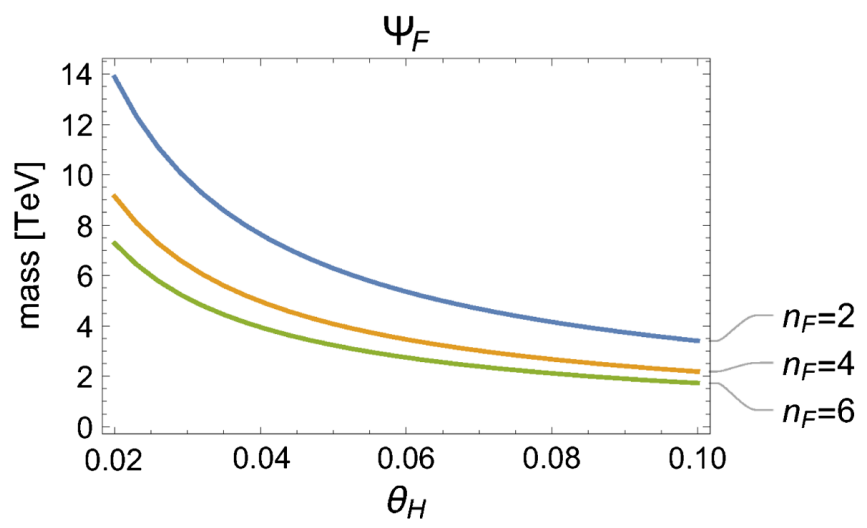

FIG. 5. $\theta_{H}$ and $n_{F}$ dependence of the mass of the dark fermion $\Psi_{F}$. Here $z_{L}=10^{10}, n_{V}=2$, and $c_{V}=0.2$. fermions have relatively small masses compared with the KK mass scale $m_{\mathrm{KK}}$. The lightest neutral component of the dark fermions can be a candidate for dark matter.

In Fig. 5 the mass of $\Psi_{F}$ is plotted as a function of $\theta_{H}$ for several $n_{F}$. The mass decreases as $n_{F}$ increases. Similar behavior is obtained for $\Psi_{(1,5)}^{ \pm}$as $n_{V}$ is varied with $c_{V}$ fixed.

\section{SUMMARY}

In this paper we have examined the effective potential $V_{\text {eff }}\left(\theta_{H}\right)$ in GUT-inspired $S O(5) \times U(1) \times S U(3)$ gaugeHiggs unification to confirm that electroweak symmetry breaking is dynamically induced by the Hosotani mechanism. From $V_{\text {eff }}\left(\theta_{H}\right)$ the cubic and quartic self-couplings, $\lambda_{3}$ and $\lambda_{4}$, of the Higgs boson have been determined. We have shown the $\theta_{H}$ universality of these couplings, i.e., they are determined as functions of $\theta_{H}$ to high accuracy, irrespective of the details of other parameters in the theory. For $\theta_{H}=0.1(0.15), \lambda_{3}$ and $\lambda_{4}$ are smaller than those in the standard model by $7.7 \%(8.1 \%)$ and $30 \%$ (32\%), respectively. The $\theta_{H}$ universality in $\lambda_{3}$ and $\lambda_{4}$ is understood as a result of the factorization property of each component in the contributions to the effective potential, which is valid to high accuracy in the Randall-Sundrum warped space with $z_{L} \gg 1$.

The $\theta_{H}$ universality gives the model great predictive power. Once the value of $\theta_{H}$ is determined by experimental data, many other physical quantities such as the masses and couplings of various particles can be predicted. It has been known that gauge-Higgs unification models in the RS space predict large parity violation in the couplings of quarks and leptons to $Z^{\prime}$ particles (KK modes of $\gamma, Z$, and $Z_{R}$ ). This effect can be clearly seen in electron-positron collision experiments with polarized electron/positron beams in which $\theta_{H}$ is the most important parameter. $Z^{\prime}$ particles can be directly produced at the LHC, and parityviolating couplings would manifest in the rapidity distribution in $t \bar{t}$ production. CKM mixing with natural FCNC suppression is also incorporated in the GUT-inspired gauge-Higgs unification. It would be interesting to pin down the behavior of the model at finite temperature and its implications for cosmology. $S O(5) \times U(1) \times S U(3)$ gauge-Higgs unification is one of the most promising scenarios beyond the standard model. We shall come back to these issues in the future.

\section{ACKNOWLEDGMENTS}

This work was supported in part by the European Regional Development Fund-Project Engineering Applications of Microworld Physics (No. CZ.02.1.01/0.0/0.0/ 16_019/0000766) (Y.O.), by the National Natural Science Foundation of China (Grant Nos. 11775092, 11675061, 11521064, 11435003 and 11947213) (S. F.), by the International Postdoctoral Exchange Fellowship Program (IPEFP) (S. F.), and by the Japan Society for 
the Promotion of Science, Grants-in-Aid for Scientific Research, No. 19K03873 (Y.H.) and Nos. 18H05543 and 19K23440 (N. Y.).

\section{APPENDIX A: MASS SPECTRUM}

In evaluating the effective potential $V_{\text {eff }}\left(\theta_{H}\right)$ in Sec. III, one needs to know the mass spectrum of each KK tower of the fields in the model. It is sufficient to know the form of functions whose zeros determine the mass spectrum. These functions have been given in Ref. [16]. We summarize them in this appendix for convenience.

We first introduce

$$
F_{\alpha, \beta}(u, v) \equiv J_{\alpha}(u) Y_{\beta}(v)-Y_{\alpha}(u) J_{\beta}(v),
$$

where $J_{\alpha}(u)$ and $Y_{\alpha}(u)$ are Bessel functions of the first and second kind. For gauge fields we define

$$
\begin{aligned}
C(z ; \lambda) & =\frac{\pi}{2} \lambda z z_{L} F_{1,0}\left(\lambda z, \lambda z_{L}\right), \\
S(z ; \lambda) & =-\frac{\pi}{2} \lambda z F_{1,1}\left(\lambda z, \lambda z_{L}\right), \\
C^{\prime}(z ; \lambda) & =\frac{\pi}{2} \lambda^{2} z z_{L} F_{0,0}\left(\lambda z, \lambda z_{L}\right), \\
S^{\prime}(z ; \lambda) & =-\frac{\pi}{2} \lambda^{2} z F_{1,1}\left(\lambda z, \lambda z_{L}\right) .
\end{aligned}
$$

For fermion fields with a bulk mass parameter $c$, we define

$$
\begin{aligned}
& \left(\begin{array}{c}
C_{L} \\
S_{L}
\end{array}\right)(z ; \lambda, c)= \pm \frac{\pi}{2} \lambda \sqrt{z z_{L}} F_{c+\frac{1}{2}, c \mp \frac{1}{2}}\left(\lambda z, \lambda z_{L}\right), \\
& \left(\begin{array}{c}
C_{R} \\
S_{R}
\end{array}\right)(z ; \lambda, c)=\mp \frac{\pi}{2} \lambda \sqrt{z z_{L}} F_{c-\frac{1}{2}, c \pm \frac{1}{2}}\left(\lambda z, \lambda z_{L}\right),
\end{aligned}
$$

and

$$
\begin{aligned}
& \mathcal{C}_{R 1}(z ; \lambda, c, \tilde{m})=C_{R}(z ; \lambda, c+\tilde{m})+C_{R}(z ; \lambda, c-\tilde{m}), \\
& \mathcal{C}_{R 2}(z ; \lambda, c, \tilde{m})=S_{R}(z ; \lambda, c+\tilde{m})-S_{R}(z ; \lambda, c-\tilde{m}), \\
& \mathcal{S}_{L 1}(z ; \lambda, c, \tilde{m})=S_{L}(z ; \lambda, c+\tilde{m})+S_{L}(z ; \lambda, c-\tilde{m}), \\
& \mathcal{S}_{L 2}(z ; \lambda, c, \tilde{m})=C_{L}(z ; \lambda, c+\tilde{m})-C_{L}(z ; \lambda, c-\tilde{m}), \\
& \mathcal{C}_{L 1}(z ; \lambda, c, \tilde{m})=C_{L}(z ; \lambda, c+\tilde{m})+C_{L}(z ; \lambda, c-\tilde{m}), \\
& \mathcal{C}_{L 2}(z ; \lambda, c, \tilde{m})=S_{L}(z ; \lambda, c+\tilde{m})-S_{L}(z ; \lambda, c-\tilde{m}), \\
& \mathcal{S}_{R 1}(z ; \lambda, c, \tilde{m})=S_{R}(z ; \lambda, c+\tilde{m})+S_{R}(z ; \lambda, c-\tilde{m}), \\
& \mathcal{S}_{R 2}(z ; \lambda, c, \tilde{m})=C_{R}(z ; \lambda, c+\tilde{m})-C_{R}(z ; \lambda, c-\tilde{m}) .
\end{aligned}
$$

\section{Gauge bosons}

The mass spectrum $\left\{m_{n}=k \lambda_{n}\right\}$ of $W$ and $W_{R}$ towers is determined by
$W$ tower: $2 S(1 ; \lambda) C^{\prime}(1 ; \lambda)+\lambda \sin ^{2} \theta_{H}=0$,

$W_{R}$ tower: $C(1 ; \lambda)=0$.

The spectrum of $\gamma, Z, Z_{R}$, and $A_{z}$ towers is determined by

$\gamma$ tower: $C^{\prime}(1 ; \lambda)=0$,

$Z$ tower: $2 S(1 ; \lambda) C^{\prime}(1 ; \lambda)+\left(1+s_{\phi}^{2}\right) \lambda \sin ^{2} \theta_{H}=0$,

$Z_{R}$ tower: $C(1 ; \lambda)=0$,

$A_{z}$ tower: $S(1 ; \lambda) C^{\prime}(1 ; \lambda)+\lambda \sin ^{2} \theta_{H}=0$.

\section{Fermions}

With given up-type quark masses $m_{Q}=\left(m_{u}, m_{c}, m_{t}\right)$ the bulk mass parameter $c_{Q}=\left(c_{u}, c_{c}, c_{t}\right)$ of up-type quark multiplets is fixed by

$$
S_{L}\left(1 ; \lambda, c_{Q}\right) S_{R}\left(1 ; \lambda, c_{Q}\right)+\sin ^{2} \frac{\theta_{H}}{2}=0,
$$

where $\lambda=\lambda_{Q}=m_{Q} / k$. Then the spectrum of up-type quark towers is determined by Eq. (A7). In the down-type quark sector there are brane interactions which mix $d^{\prime \alpha}$ and $D^{+\beta}$ through Eq. (2.14). When brane interactions are diagonal in the generation space, $\mu^{\alpha \beta}=\delta^{\alpha \beta} \mu_{\alpha}$, the spectrum of down-type quark towers is determined by

$$
\begin{aligned}
& \left(S_{L}^{Q} S_{R}^{Q}+\sin ^{2} \frac{\theta_{H}}{2}\right)\left(\mathcal{S}_{L 1}^{D} \mathcal{S}_{R 1}^{D}-\mathcal{S}_{L 2}^{D} \mathcal{S}_{R 2}^{D}\right) \\
& \quad+\left|\mu_{1}\right|^{2} C_{R}^{Q} S_{R}^{Q}\left(\mathcal{S}_{L 1}^{D} \mathcal{C}_{L 1}^{D}-\mathcal{S}_{L 2}^{D} \mathcal{C}_{L 2}^{D}\right)=0 \\
& S_{L}^{Q}=S_{L}\left(1 ; \lambda, c_{Q}\right), \mathcal{S}_{L 1}^{D}=\mathcal{S}_{L 1}\left(1 ; \lambda, c_{D}, \tilde{m}_{D}\right), \quad \text { etc. }
\end{aligned}
$$

where $\mu_{1}=\left(\mu_{d}, \mu_{s}, \mu_{b}\right), c_{D}=\left(c_{D_{d}}, c_{D_{s}}, c_{D_{b}}\right)$, and $\tilde{m}_{D}=$ $\left(\tilde{m}_{D_{d}}, \tilde{m}_{D_{s}}, \tilde{m}_{D_{b}}\right)$. The parameters $\mu_{1}, c_{D}, \tilde{m}_{D}$ are determined such that $\lambda=\left(\lambda_{d}, \lambda_{s}, \lambda_{b}\right)=k^{-1}\left(m_{d}, m_{s}, m_{b}\right)$ solves Eq. (A8) in each generation. For the third generation, for instance, we take $\left(\mu_{b}, c_{D_{b}}, \tilde{m}_{D_{d}}\right)=(0.1,1.044,1.0)$. Only the top-quark multiplet among quark multiplets gives a relevant contribution to $V_{\text {eff }}\left(\theta_{H}\right)$. By considering general $\mu^{\alpha \beta}$ the CKM mixing is incorporated with natural FCNC suppression [20].

With given charged lepton masses $m_{L}=\left(m_{e}, m_{\mu}, m_{\tau}\right)$ the bulk mass parameter $c_{L}=\left(c_{e}, c_{\mu}, c_{\tau}\right)$ of charged lepton multiplets is fixed by

$$
S_{L}\left(1 ; \lambda, c_{L}\right) S_{R}\left(1 ; \lambda, c_{L}\right)+\sin ^{2} \frac{\theta_{H}}{2}=0,
$$

where $\lambda=\lambda_{L}=m_{L} / k$. Then the spectrum of charged lepton towers is determined by Eq. (A9). In the neutrino sector brane interactions $\operatorname{mix} \nu^{\alpha}, \nu^{\prime \alpha}, \chi^{\alpha}$. When both 
$M^{\alpha \beta}=\delta^{\alpha \beta} M_{\alpha}$ in Eq. (2.10) and $m_{B}^{\alpha \beta}=\delta^{\alpha \beta} m_{B}^{\alpha}$ in Eq. (2.15) are diagonal, the spectrum of neutrino towers is determined by

$(k \lambda-M)\left(S_{L}^{L} S_{R}^{L}+\sin ^{2} \frac{\theta_{H}}{2}\right)+\frac{m_{B}^{2}}{k} S_{R}^{L} C_{R}^{L}=0$,

$S_{R}^{L}=S_{R}\left(1 ; \lambda, c_{L}\right), \quad$ etc.

where $M=\left(M_{1}, M_{2}, M_{3}\right)$ and $m_{B}=\left(m_{B}^{1}, m_{B}^{2}, m_{B}^{3}\right)$. With $c_{L}<-\frac{1}{2}$ the light neutrino mass is given by

$$
m_{\nu} \sim \frac{m_{L}^{2} M}{\left(2\left|c_{L}\right|-1\right) m_{B}^{2}}
$$

in each generation. Contributions from lepton multiplets to $V_{\text {eff }}\left(\theta_{H}\right)$ are negligible.

The spectrum of dark fermion $\Psi_{F}$ towers is determined by

$$
S_{L}\left(1 ; \lambda, c_{F}\right) S_{R}\left(1 ; \lambda, c_{F}\right)+\cos ^{2} \frac{\theta_{H}}{2}=0 .
$$

The spectrum of charged components of dark fermion $\Psi_{(1,5)}^{ \pm}$towers is determined by

$\mathcal{S}_{L 1}\left(1 ; \lambda, c_{V}\right) \mathcal{S}_{R 1}\left(1 ; \lambda, c_{V}\right)-\mathcal{S}_{L 2}\left(1 ; \lambda, c_{V}\right) \mathcal{S}_{R 2}\left(1 ; \lambda, c_{V}\right)=0$,

whereas the spectrum of neutral component towers is determined by

$$
\begin{aligned}
& \left\{B_{0}\left(\lambda, c_{V}, \tilde{m}_{V}\right)-2 \cos 2 \theta_{H}\right\}^{2}=0 \\
& B_{0}(\lambda, c, \tilde{m})= \\
& \quad C_{L}(1 ; \lambda, c+\tilde{m}) C_{R}(1 ; \lambda, c-\tilde{m})+C_{L}(1 ; \lambda, c-\tilde{m}) C_{R}(1 ; \lambda, c+\tilde{m}) \\
& \quad+S_{L}(1 ; \lambda, c+\tilde{m}) S_{R}(1 ; \lambda, c-\tilde{m})+S_{L}(1 ; \lambda, c-\tilde{m}) S_{R}(1 ; \lambda, c+\tilde{m}) .
\end{aligned}
$$

There are two degenerate towers. ${ }^{1}$

\section{APPENDIX B: USEFUL FUNCTIONS}

As shown in the formula (3.3), a mass-determining function $\rho\left(m ; \theta_{H}\right)$ is analytically continued to $\rho\left(i y ; \theta_{H}\right)$. We summarize the functions used in the evaluation of $V_{\text {eff }}\left(\theta_{H}\right)$ in Sec. III. We introduce

$$
\hat{F}_{\alpha, \beta}(u, v) \equiv I_{\alpha}(u) K_{\beta}(v)-e^{-i(\alpha-\beta) \pi} K_{\alpha}(u) I_{\beta}(v),
$$

where $I_{\alpha}(u)$ and $K_{\alpha}(u)$ are modified Bessel functions of the first and second kind. In terms of $\hat{F}_{\alpha, \beta}(u, v)$ we define

$$
\begin{aligned}
\hat{C}(q) & =q \hat{F}_{1,0}\left(q z_{L}^{-1}, q\right), \\
\hat{S}(q) & =i q z_{L}^{-1} \hat{F}_{1,1}\left(q z_{L}^{-1}, q\right), \\
\hat{C}^{\prime}(q) & =q^{2} z_{L}^{-1} \hat{F}_{0,0}\left(q z_{L}^{-1}, q\right), \\
\hat{S}^{\prime}(q) & =-i q^{2} z_{L}^{-2} \hat{F}_{1,1}\left(q z_{L}^{-1}, q\right)
\end{aligned}
$$

for gauge fields. For fermion fields with $c>0$ we define

$$
\begin{aligned}
& \hat{C}_{L}(q ; c)=q z_{L}^{-1 / 2} \hat{F}_{c+\frac{1}{2}, c-\frac{1}{2}}\left(q z_{L}^{-1}, q\right), \\
& \hat{S}_{L}(q ; c)=i q z_{L}^{-1 / 2} \hat{F}_{c+\frac{1}{2}, c+\frac{1}{2}}\left(q z_{L}^{-1}, q\right), \\
& \hat{C}_{R}(q ; c)=q z_{L}^{-1 / 2} \hat{F}_{c-\frac{1}{2}, c+\frac{1}{2}}\left(q z_{L}^{-1}, q\right), \\
& \hat{S}_{R}(q ; c)=-i q z_{L}^{-1 / 2} \hat{F}_{c-\frac{1}{2}, c-\frac{1}{2}}\left(q z_{L}^{-1}, q\right) .
\end{aligned}
$$

For $c<0$, we use the relations

$$
\hat{C}_{L}(q ;-c)=\hat{C}_{R}(q ; c), \hat{S}_{L}(q ;-c)=-\hat{S}_{R}(q ; c) .
$$

\footnotetext{
${ }^{1}$ There was a typo in Eq. (D.16) of Ref. [16]. The last term in the second line, $s_{H}^{2} c_{H}^{2}\left(\mathcal{C}_{R 1}^{V} \mathcal{C}_{L 2}^{V}-\mathcal{C}_{R 2}^{V} \mathcal{S}_{L 1}^{V}\right)^{2}$, should be $s_{H}^{2} c_{H}^{2}\left(\mathcal{C}_{R 1}^{V} \mathcal{S}_{L 2}^{V}-\mathcal{C}_{R 2}^{V} \mathcal{S}_{L 1}^{V}\right)^{2}$. With this correction, Eq. (D.16) of Ref. [16] coincides with Eq. (A14) in the current paper.
} 
[1] Y. Hosotani, Dynamical mass generation by compact extra dimensions, Phys. Lett. B 126, 309 (1983).

[2] Y. Hosotani, Dynamics of nonintegrable phases and gauge symmetry breaking, Ann. Phys. (N.Y.) 190, 233 (1989).

[3] A. T. Davies and A. McLachlan, Gauge group breaking by Wilson loops, Phys. Lett. B 200, 305 (1988); Congruency class effects in the Hosotani model, Nucl. Phys. B317, 237 (1989).

[4] H. Hatanaka, T. Inami, and C. S. Lim, The gauge hierarchy problem and higher dimensional gauge theories, Mod. Phys. Lett. A 13, 2601 (1998).

[5] H. Hatanaka, Matter representations and gauge symmetry breaking via compactified space, Prog. Theor. Phys. 102, 407 (1999).

[6] M. Kubo, C. S. Lim, and H. Yamashita, The Hosotani mechanism in bulk gauge theories with an orbifold extra space $S^{1} / Z_{2}$, Mod. Phys. Lett. A 17, 2249 (2002).

[7] A. Pomarol and M. Quiros, The Standard Model from extra dimensions, Phys. Lett. B 438, 255 (1998).

[8] C. A. Scrucca, M. Serone, and L. Silvestrini, Electroweak symmetry breaking and fermion masses from extra dimensions, Nucl. Phys. B669, 128 (2003).

[9] K. Agashe, R. Contino, and A. Pomarol, The minimal composite Higgs model, Nucl. Phys. B719, 165 (2005).

[10] G. Cacciapaglia, C. Csaki, and S. C. Park, Fully radiative electroweak symmetry breaking, J. High Energy Phys. 03 (2006) 099.

[11] A. D. Medina, N. R. Shah, and C. E. M. Wagner, GaugeHiggs unification and radiative electroweak symmetry breaking in warped extra dimensions, Phys. Rev. D 76, 095010 (2007).

[12] Y. Hosotani, K. Oda, T. Ohnuma, and Y. Sakamura, Dynamical electroweak symmetry breaking in $S O(5) \times$ $U(1)$ gauge-Higgs unification with top and bottom quarks, Phys. Rev. D 78, 096002 (2008); Erratum, Phys. Rev. D 79, 079902 (2009).

[13] S. Funatsu, H. Hatanaka, Y. Hosotani, Y. Orikasa, and T. Shimotani, Novel universality and Higgs decay $H \rightarrow \gamma \gamma, g g$ in the $S O(5) \times U(1)$ gauge-Higgs unification, Phys. Lett. B 722, 94 (2013).

[14] S. Funatsu, H. Hatanaka, Y. Hosotani, and Y. Orikasa, Distinct signals of the gauge-Higgs unification in $e^{+} e^{-}$ collider experiments, Phys. Lett. B 775, 297 (2017).

[15] Y. Adachi and N. Maru, Revisiting electroweak symmetry breaking and the Higgs boson mass in gauge-Higgs unification, Phys. Rev. D 98, 015022 (2018).

[16] S. Funatsu, H. Hatanaka, Y. Hosotani, Y. Orikasa, and N. Yamatsu, GUT inspired $S O(5) \times U(1) \times S U(3)$ gaugeHiggs unification, Phys. Rev. D 99, 095010 (2019).

[17] J. Yoon and M.E. Peskin, Fermion pair production in $S O(5) \times U(1)$ gauge-Higgs unification models, arXiv: 1811.07877 .

[18] J. Yoon and M. E. Peskin, Dissection of an $S O(5) \times U(1)$ gauge-Higgs unification model, Phys. Rev. D 100, 015001 (2019).

[19] S. Funatsu, Forward-backward asymmetry in the gaugeHiggs unification at the International Linear Collider, Eur. Phys. J. C 79, 854 (2019).

[20] S. Funatsu, H. Hatanaka, Y. Hosotani, Y. Orikasa, and N. Yamatsu, CKM matrix and FCNC suppression in
$S O(5) \times U(1) \times S U(3)$ gauge-Higgs unification, Phys. Rev. D 101, 055016 (2020).

[21] S. Bilokin, R. Pöschl, and F. Richard, Measurement of b quark EW couplings at ILC, arXiv:1709.04289; F. Richard, Bhabha scattering at ILC250, arXiv:1804.02846; A. Irles, R. Pöschl, F. Richard, and H. Yamamoto, Complementarity between ILC250 and ILC-GigaZ, arXiv:1905.00220; A. Irles, R. Pöschl, and F. Richard, Production and measurement of $e^{+} e^{-} \rightarrow c \bar{c}$ signatures at the $250 \mathrm{GeV}$ ILC, arXiv: 2002.05805 .

[22] K. Fujii et al., Physics case for the $250 \mathrm{GeV}$ stage of the International Linear Collider, arXiv:1710.07621; H. Aihara et al. (ILC Collaboration), The International Linear Collider. A global project, arXiv:1901.09829; P. Bambade et al., The International Linear Collider. A Global project, arXiv:1903.01629.

[23] G. Burdman and Y. Nomura, Unification of Higgs and gauge fields in five dimensions, Nucl. Phys. B656, 3 (2003); N. Haba, Masatomi Harada, Y. Hosotani, and Y. Kawamura, Dynamical rearrangement of gauge symmetry on the orbifold $S^{1} / Z_{2}$, Nucl. Phys. B657, 169 (2003); Erratum, Nucl. Phys. B669, 381 (2003); N. Haba, Y. Hosotani, Y. Kawamura, and T. Yamashita, Dynamical symmetry breaking in gauge Higgs unification on orbifold, Phys. Rev. D 70, 015010 (2004); C. S. Lim and N. Maru, Towards a realistic grand gauge-Higgs unification, Phys. Lett. B 653, 320 (2007); K. Kojima, K. Takenaga, and T. Yamashita, Grand gauge-Higgs unification, Phys. Rev. D 84, 051701(R) (2011); Gauge symmetry breaking patterns in an SU(5) grand gauge-Higgs unification, Phys. Rev. D 95, 015021 (2017); M. Frigerio, J. Serra, and A. Varagnolo, Composite GUTs: Models and expectations at the LHC, J. High Energy Phys. 06 (2011) 029; M. Kakizaki, S. Kanemura, H. Taniguchi, and T. Yamashita, Higgs sector as a probe of supersymmetric grand unification with the Hosotani mechanism, Phys. Rev. D 89, 075013 (2014); N. Maru and Y. Yatagai, Fermion mass hierarchy in grand gauge-Higgs unification, Prog. Theor. Exp. Phys. (2019), 083B03.

[24] Y. Hosotani and N. Yamatsu, Gauge-Higgs grand unification, Prog. Theor. Exp. Phys. (2015), 111B01.

[25] A. Furui, Y. Hosotani, and N. Yamatsu, Toward realistic gauge-Higgs grand unification, Prog. Theor. Exp. Phys. (2016), 093B01; Y. Hosotani and N. Yamatsu, Electroweak symmetry breaking and mass spectra in six-dimensional gauge-Higgs grand unification, Prog. Theor. Exp. Phys. (2018), 023B05.

[26] Y. Hosotani and N. Yamatsu, Gauge-Higgs seesaw mechanism in 6-dimensional grand unification, Prog. Theor. Exp. Phys. (2017), 091B01.

[27] C. Englert, D. J. Miller, and D. D. Smaranda, Phenomenology of GUT-inspired gauge-Higgs unification, Phys. Lett. B 802, 135261 (2020).

[28] Y. Adachi and N. Maru, Strong first order electroweak phase transition in gauge-Higgs unification at finite temperature, Phys. Rev. D 101, 036013 (2020).

[29] G. F. Giudicea, C. Grojean, A. Pomarol, and R. Rattazzi, The strongly-interacting light Higgs, J. High Energy Phys. 06 (2007) 045; R. Contino, C. Grojean, M. Moretti, F. Piccinini, and R. Rattazzi, Strong double Higgs production at the LHC, J. High Energy Phys. 05 (2010) 089; 
R. Contino, M. Ghezzi, M. Moretti, G. Panico, F. Piccinini, and A. Wulzer, Anomalous couplings in double Higgs production, J. High Energy Phys. 08 (2012) 154; R. Contino, M. Ghezzi, C. Grojean, M. Muhlleitner, and M. Spira, Effective Lagrangian for a light Higgs-like scalar, J. High Energy Phys. 07 (2013) 035.

[30] G. Cacciapaglia, C. Pica, and F. Sannino, Fundamental composite dynamics: A review, arXiv:2002.04914; R. Contino, The Higgs as a composite Nambu-Goldstone Boson, TASI (2009) 235 [arXiv:1005.4269]; B. Bellazzini, C. Csaki, and J. Serra, Composite Higgses, Eur. Phys. J. C 74, 2766 (2014).

[31] S. Funatsu, H. Hatanaka, Y. Hosotani, Y. Orikasa, and T. Shimotani, LHC signals of the $S O(5) \times U(1)$ gauge-Higgs unification, Phys. Rev. D 89, 095019 (2014); S. Funatsu, H. Hatanaka, Y. Hosotani, and Y. Orikasa, Collider signals of $W^{\prime}$ and $Z^{\prime}$ bosons in the gauge-Higgs unification, Phys. Rev. D 95, 035032 (2017).
[32] L. Randall and R. Sundrum, A Large Mass Hierarchy from a Small Extra Dimension, Phys. Rev. Lett. 83, 3370 (1999).

[33] T. Gherghetta and A. Pomarol, Bulk fields and supersymmetry in a slice of AdS, Nucl. Phys. B586, 141 (2000).

[34] M. Tanabashi et al. (Particle Data Group), Review of particle physics, Phys. Rev. D 98, 030001 (2018), Chap. 10. Electroweak model and constraints on new physics.

[35] A. Falkowski, Holographic pseudo-Goldstone boson, Phys. Rev. D 75, 025017 (2007).

[36] Y. Hosotani, M. Tanaka, and N. Uekusa, $H$ parity and the stable Higgs boson in the $S O(5) \times U(1)$ gauge-Higgs unification, Phys. Rev. D 82, 115024 (2010).

[37] Y. Hosotani and Y. Kobayashi, Yukawa couplings and effective interactions in gauge-Higgs unification, Phys. Lett. B 674, 192 (2009).

[38] Y. Hosotani, Gauge-Higgs unification-Beyond the Standard Model, in SGC Library -143 (SAIENSU-SHA Co., Ltd, Tokyo, 2018), Section 8.1 (in Japanese). 